Études 



\title{
Polynormativité et contrôle social du monde des affaires : l'exemple de l'interventionnisme et de la punitivité des juges du Tribunal de commerce de Paris
}

\author{
Emmanuel Lazega *, Lise Mounier ** \\ * IRISES/CERSO - CNRS, Université Paris IX-Dauphine, Place du Maréchal de Lattre de Tassigny, F-75775 Paris Cedex 16 \\ <emmanuel.lazega@dauphine.fr>. \\ ** Centre Maurice Halbwachs - CNRS, École Normale Supérieure, 48 boulevard Jourdan, F-75014 Paris. \\ $<$ lise.mounier@ens.fr>
}

Résumé

Cet article explore la polynormativité contemporaine du contrôle social des marchés en la reliant à l'origine professionnelle des 151 juges consulaires du Tribunal de commerce de Paris (2005-2006). Le commentaire jurisprudentiel des juges sur trois cas d'espèce où ils disposent d'un pouvoir souverain d'appréciation met au jour des catégories pratiques de jugement en matière économique, notamment des variations intéressantes en matière d'interventionnisme et de punitivité dans les conflits caractérisant le fonctionnement des marchés et la gouvernance d'entreprise. De forts contrastes apparaissent dans ces domaines, par exemple, entre les juges issus du milieu de la banque-finance et ceux issus du secteur du bâtiment et travaux publics.

Interventionnisme - Juges consulaires - Néo-corporatismes - Polynormativité - Punitivité - Tribunal de commerce.

Summary Polynormativity and Social Control of Business: Interventionism and Punitivity of Lay Judges at the Commercial Court of Paris

This paper explores the contemporary polynormativity of the social control of business. We look at the relationship between the professional origin of 151 lay judges at the Commercial Court of Paris (2005-2006) and the norms on which they rely to make discretionary judicial decisions. The judges' work is approached indirectly, based on interviews managed as jurisprudential discussion of three specific cases involving damages awarded in unfair competition, breach of contract, and conflict between minority and majority shareholders. Firstly, variations are identified in the judges' punitivity and interventionism with regard to conflicts in markets and in corporate governance. Secondly, strong differences emerge, for example, between judges coming from the financial industry and those from the building sector.

Commercial court - Interventionism - Lay judges - Neo-corporatism Polynormativity - Punitivity - Social control of business. 


\section{Introduction}

Dans un monde globalisé, les économies de marché sont caractérisées par la coexistence de plusieurs types de normes visant la régulation de la conduite des acteurs du monde des affaires et la résolution des conflits entre eux : les normes promulguées directement par les États-Nations ou par des institutions auxquelles ces États-Nations délèguent des pouvoirs (comme l'OMC) ; et les normes formulées par les acteurs privés eux-mêmes, en particulier par les entreprises et leurs institutions semi-publiques comme les tribunaux d'arbitrage. Les spécialistes de ces normes privées affirment qu'une nouvelle lex mercatoria contemporaine et internationale serait en gestation depuis deux générations ${ }^{1}$, c'est-à-dire un ensemble de règles à la fois formelles et informelles sur lesquelles s'appuie la marchandisation croissante des sociétés contemporaines à l'échelle globale.

La coexistence de ces deux types de normes, que nous appelons «polynormativité " du monde des affaires, pose souvent problème ${ }^{2}$. Parmi ces principaux problèmes "génériques", on peut relever ceux de la légitimité démocratique, de la qualité ou de la mise en œuvre des règles privées. Ces dernières, formulées par des acteurs économiques souvent plus soucieux de gestion des risques que d'état de droit, engendrent à leur tour des pressions de type bottom-up sur les systèmes juridiques officiels, nationaux et internationaux. Leur connaissance est donc importante.

L'un des problèmes les plus frappants qui se pose au sujet de ces règles privées nous semble être, en amont de ces grands et principaux problèmes "génériques ", le manque de connaissances scientifiques et publiques des formes que prend cette polynormativité. Nous proposons dans cet article une étude très exploratoire d'une dimension peu connue de cette polynormativité, à savoir les luttes entre normes privées et informelles issues du monde des affaires, et sur lesquelles ce dernier s'appuie pour la résolution des conflits entre entreprises ou entre parties prenantes dans l'entreprise et les marchés. Nous approchons cette dimension peu connue, paradoxalement, par l'examen du fonctionnement d'une institution consulaire française, chargée de la mise en œuvre des règles de droit étatiques, mais dont les membres, les juges consulaires, sont des représentants du monde des affaires qui valorisent aussi explicitement les normes privées.

L'émergence de ces normes privées ne relève pas d'un processus politique au sens institutionnel du terme, dans la mesure où - du fait de la séparation des pouvoirs législatifs et judiciaires - les acteurs privés n'ont pas la capacité de formuler des normes officielles indépendamment des autorités publiques, même si les juges consulaires aiment à rappeler que les usages sont sources de droit. Ce processus

1. Klaus Peter Berger, The Creeping Codification of the Lex Mercatoria, La Haye, Kluwer Law International, 1999 ; Harold J. BERMAN, « Mercantile Law », in ID., Law and Revolution: The Formation of the Western Legal Tradition, Cambridge (Mass.), Harvard University Press, 1983, p. 333-356 ; Filip DE Ly, International Business Law and Lex Mercatoria, Amsterdam, North-Holland, 1992.

2. Ian Ayres et John BraithWAITE, Responsive Regulation: Transcending the Deregulation Debate, Oxford, Oxford University Press, 1992. 
n'en est pas simple pour autant. Il s'apparente en partie à une lutte entre normes privées concurrentes pour la diffusion - dans des institutions publiques ou privées - de solutions différentes à apporter à ces conflits. Ce processus de formulation des règles privées commence, chez ces acteurs, par la création de représentations et par la promotion de raisonnements pratiques basés sur ces représentations.

Là où Durkheim, dans sa préface à la $2^{\mathrm{e}}$ édition de De la division du travail social, voyait dans la production des normes corporatistes une réponse possible à ce qu'il considérait comme l'anomie du secteur économique, nous cherchons à mettre davantage au jour la polynormativité de ce système complexe. Cette polynormativité recouvre en fait un conflit entre de nombreuses normes (polynomie) plutôt que l'absence de normes (anomie).

Pour cela, nous nous appuyons sur une étude de cas, celle du fonctionnement d'une institution consulaire, le Tribunal de commerce de Paris (TCP). Le tribunal de commerce est une juridiction - comme les conseils de prud'hommes pour les conflits du travail - construite à partir d'un espace social localisé. Depuis le Xvi siècle, c'est elle qui a reçu de l'État le mandat de régler les conflits entre les commerçants. Elle représente une forme originale de partage des coûts du contrôle car les juges consulaires sont des hommes et des femmes d'affaires «bénévoles » : l'État n'a donc pas à les rémunérer comme des juges fonctionnaires ou de carrière, le coût salarial de la justice étant reporté sur les juges eux-mêmes lorsqu'ils ne sont pas (ou plus) salariés, ou sur les entreprises dont ils sont les salariés. Dans cette solution institutionnelle fondée sur l'existence d'institutions intermédiaires (de statut public, mais dont les membres sont issus du monde des affaires), les coûts du contrôle sont donc partagés par l'État, les entreprises et les juges individuels qui se considèrent comme des représentants de la société civile.

L'histoire montre que le travail de « régulation » et de contrôle social du monde des affaires engage nécessairement la relation de ce dernier avec l'État. Le monde des affaires a très tôt participé à la gouvernance de ses marchés ainsi qu'au partage des coûts du contrôle social de ses activités économiques. En Europe, c'est lui qui a d'abord créé ses propres régulations. Mais il a très tôt recouru à l'État, dans un rapport complexe, pour obtenir de meilleurs moyens d'officialisation et de sanction de ses normes.

Au cours des $\mathrm{XI}^{\mathrm{e}}$ et XII ${ }^{\mathrm{e}}$ siècles, d'abord dans les cités-États qu'ils dominaient, les commerçants européens développèrent leur propre droit commercial, qui en vint à coexister avec d'autres droits, comme le droit canonique. L'achat, la vente, le transport, l'assurance étaient régulés par un droit issu des corporations marchandes, que l'on a pu appeler, en raccourci, la lex mercatoria européenne ${ }^{3}$ car, malgré des variantes locales, de grands principes se retrouvaient de place de commerce en place de commerce. On a ainsi pu dire que la lex mercatoria du Moyen-Âge a été le

3. Harold J. Berman, Law and Revolution: The Formation of the Western Legal Tradition, Cambridge (Mass.), Harvard University Press, 1983. 
fondement juridique du capitalisme ${ }^{4}$. Les marchands, qui constituaient un groupe social relativement distinct des autres et aux intérêts économiques bien spécifiés, avaient leurs propres tribunaux et élisaient des juges parmi leurs pairs (comme les artisans le faisaient dans les guildes, métier par métier, mais dans un regroupement plus large de tous les types de commerce sur une base locale). On y délivrait une justice rapide sans argumentation juridique sophistiquée, fondée en équité, sans avocats professionnels. Les commerçants contrôlaient ce qui se passait dans leurs marchés et foires, mais, parallèlement, ils faisaient souvent appel au pouvoir d'État pour mettre en œuvre les décisions qu'ils prenaient - ce qui ne signifie pas qu'ils ne pouvaient pas, dans certaines circonstances, compter seulement sur d'autres moyens de contrainte comme la réputation ${ }^{5}$.

Cette lex mercatoria évolua ainsi différemment selon les pays, en fonction de leurs types d'État (plus ou moins absolutiste) et de droit (entre common law et codification). En France en particulier, c'est une forme spécifique d'institution de régulation conjointe qui en émergea à partir $\mathrm{du} \mathrm{XvI}^{\mathrm{e}}$ siècle : les tribunaux de commerce. Ceux-ci apparaissent comme un compromis visant à préserver les avantages des deux formes de régulation, endogène et exogène : tout en conservant les principes de la lex mercatoria (juges commerçants, justice rapide et peu chère, respect des usages du commerce), ils sont intégrés dans l'administration judiciaire (bénéficiant de ses pouvoirs de sanction) et appliquent très tôt (dès la fin du XVII siècle) des lois commerciales générales. Ce compromis tient depuis le Xvi siècle, malgré des critiques régulières de son caractère hybride entre institution marchande et étatique. Ces critiques portent essentiellement sur les risques de corruption ou d'incompétence des juges ${ }^{6}$. En effet, l'un des problèmes posés par ce type d'institution est celui de l'impartialité des juges et des conflits d'intérêts auxquels ils sont confrontés. Les tribunaux ne sont pas des institutions statiques prenant des décisions atemporelles et purement rationnelles 7 . Ce sont des terrains « contestés » 8 dont le contrôle direct ou indirect fait l'objet d'une concurrence. L'approche de la

4. Max WeBER, "Zur Geschichte der Handelsgesellschaften im Mittelalter» [1889], in ID., Gesammelte Aufsätze zur Sozial- und Wirtschaftsgeschichte, Tübingen, J.C.B. Mohr, 1988, p. 312-443.

5. Paul R. Milgrom, Douglass C. North et Barry R. Weingast, The Role of Institutions in the Revival of Trade : The Law Merchant, Private Judges, and the Champagne Fairs, Economics \& Politics, 2 (1), 1990, p. 123.

6. On peut aussi se demander ce que devient ce compromis avec l'internationalisation des transactions commerciales mettant en contact des traditions juridiques diverses. Voir, au sujet de l'émergence d'une nouvelle lex mercatoria: Klaus Peter BERGER, The Creeping Codification of the Lex Mercatoria, op. cit. ; GralfPeter CALLIESS, «Reflexive Transnational Law: The Privatisation of Civil Law and the Civilization of Private Law ", Zeitschrift für Rechtssoziologie, 23(2), 2002, p. 185-216 ; Yves DEZALAY et Bryant G. GARTH, Dealing in Virtue : International Commercial Arbitration and the Construction of a Transnational Legal Order, Chicago, University of Chicago Press, 1996 ; Oliver VOLCKART et Antje MANGELS, "Are the Roots of the Modern "Lex Mercatoria” Really Medieval ? ", Southern Economic Journal, 65 (3), 1999, p. 427-450.

7. Wolf HeYDebrand et Carroll SERON, Rationalizing Justice: The Political Economy of Federal District Courts, Albany, State University of New York Press, 1990 ; Stanton WheELER, Kenneth ManN et Austin SARAT, Sitting in Judgment: The Sentencing of White Collar Criminals, New Haven, Yale University Press, 1988.

8. Au sens de Roy B. Flemming, « Contested Terrains and Regime Politics : Thinking about America's Trial Courts and Institutional Change», Law \& Social Inquiry, 23 (4), 1998, p. 941-965. Voir aussi Ana Maria FALCONI, Karima Guenfoud, Emmanuel LAZEGA, Claire LEMERCIER et Lise Mounier, «Le contrôle social du monde des affaires : une étude institutionnelle», L’Année sociologique, 55(2), 2005, p. 451-484. 
polynormativité est de ce fait indissociable d'un examen plus ou moins informé de la manière dont les intérêts organisés cherchent à contrôler à distance ce type d'institution.

Comme les magistrats de carrière, ces juges consulaires prennent aujourd'hui des décisions dans un environnement globalisé et font partie d'un système complexe et polynormatif où les normes et les sources du droit ne sont jamais entièrement cohérentes ou hiérarchiquement ordonnées entre elles. Comme toute institution judiciaire, ce tribunal doit gérer une palette de normes, valeurs ou orientations (du type softlaw) dont la relation n'est pas donnée d'avance mais résulte précisément d'un processus de prise de décision. C'est donc en approchant ce travail de prise de décision que nous contribuons à mettre au jour cette polynormativité dans le contrôle social du monde des affaires.

\section{Examen exploratoire de la polynormativité dans une institution de « régulation conjointe » : le cas du Tribunal de commerce de Paris (TCP)}

Le contrôle social du fonctionnement des marchés est exercé, dans la société française contemporaine, par de nombreuses institutions (juridictions commerciales, tribunaux d'arbitrage, autorités administratives indépendantes, etc.). Chacune de ces institutions représente une forme particulière de "régulation conjointe » du monde des affaires ${ }^{9}$. Nous entendons par "régulation conjointe " la réunion des efforts coordonnés à la fois de l'État et du monde des affaires, combinant - de manière plus ou moins conflictuelle - une régulation exogène et une auto-régulation endogène. Notre étude de la polynormativité s'appuie sur une approche organisationnelle de cette régulation conjointe. Au moins deux courants en sociologie se sont penchés sur la régulation des marchés en adoptant ce type d'approche organisationnelle. Leur objectif a été de mettre au jour soit les aspects formels et souvent exogènes de cette régulation, soit le caractère informel et souvent endogène de l'autorégulation.

Selon le premier courant, socio-juridique, ce sont les agences gouvernementales, appuyées par la justice, qui interviennent dans la régulation. Ces études examinent, entre autres, la manière dont ces agences repèrent et décident de poursuivre les entreprises qui refusent de se conformer 10. De telles mesures, qui sont souvent un compromis auquel sont arrivés les inspecteurs officiels et les dirigeants de

9. Voir à ce sujet Jean-Daniel REYNAUD, Les règles du jeu : l'action collective et et la régulation sociale, Paris, Armand Colin, 1989; Emmanuel LAZEGA, «Networks in Legal Organizations : On the Protection of Public Interest in Joint Regulation of Markets ", Wiarda Chair Inaugural Address 2003, Wiarda Institute Publications, Faculty of Law, Utrecht University, 2003 ; Emmanuel LAZEGA et Louise MounIER, «Interlocking Judges: On Joint External and Self-Governance of Markets ", in Vincent BuSKENS, Werner RauB et Chris SNIJDERS (eds.), The Governance of Relations in Markets and Organizations, Amsterdam, Boston, JAI, coll. « Research in the Sociology of Organizations », 2003, p. 267-296.

10. Ian Ayres et John BRAITHWATte, Responsive Regulation: Transcending the Deregulation Debate, op. cit.; Keith Hawkins, Environment and Enforcement: Regulation and the Social Definition of Pollution, Oxford, Oxford University Press, 1984 ; KeITH HawKIns et John M. THOMAS (eds.), Enforcing Regulation, Boston, Kluwer-Nijhof, 1984 ; Susan P. SHAPIRO, Wayward Capitalists: Target of the Securities and Exchange Commission, New Haven, Yale University Press, 1984 ; Suzanne WEAVER, Decision to Prosecute : Organization and Public Policy in the Antitrust Division, Cambridge (Mass.), MIT Press, 1977. 
l'entreprise, ne sont pas faciles à prendre, surtout lorsque cette dernière s'expose à de grandes pertes ou à des licenciements massifs, parfois même à la faillite, si la loi était appliquée à la lettre.

Le second courant explore les arrangements inter-entreprises, faisant valoir les avantages de l'autorégulation en matière de transactions inter-organisationnelles et des mécanismes informels de règlement de conflits. Et c'est justement parce que les litiges sont coûteux que les entreprises économiques préfèrent, autant que possible, régler leurs conflits de façon informelle, surtout si elles ont entretenu depuis longtemps des relations dont elles veulent protéger le caractère durable ${ }^{11}$. Les entrepreneurs essaient de donner forme à leur structure d'opportunité, d'organiser leur propre milieu et de soutenir les processus sociaux qui leur permettent de coopérer, même (ou surtout) entre concurrents ${ }^{12}$. Ici, ce qui attire l'attention, ce sont les pressions que les organisations marchandes exercent les unes sur les autres pour promouvoir une certaine conformité, pressions qui s'appuient par exemple sur la réputation et la dépendance de ressources.

Ainsi, chaque courant est axé sur un différent type d'acteurs et de système d'action susceptibles d'intervenir dans la régulation et endossant la plus grande part des coûts du contrôle: principalement l'État ou les entreprises elles-mêmes (par le biais de représentants de l'industrie ou au travers du choix de partenaires d'échanges).

En réalité, les deux systèmes de régulation ou de règlement de conflits se rejoignent à plusieurs égards, même si leur caractère " conjoint » se décline sur le mode d'un conflit plus ou moins larvé, euphémisé ou étouffé. Ayres et Braithwaite 13 en font l'illustration dans leur analyse de la « responsive self-regulation », analyse qui montre l'existence de " pyramides de mise en œuvre " (enforcement pyramids) entre les agences de réglementation de l'État et les représentants des entreprises. Ces pyramides illustrent un système de sanctions graduées où les représentants du monde des affaires et ceux de la loi peuvent utiliser des mesures de persuasion, des lettres d'avertissement, des sanctions civiles, des sanctions pénales, allant parfois jusqu'à la suspension et à la révocation de permis. Les acteurs connaissent l'existence de telles pyramides. Ils savent que chaque façon de faire respecter les contrats en appelle une autre et qu'il existe un risque d'escalade. C'est pourquoi, en

11. Stewart Macaulay, "Non-Contractual Relations in Business : A Preliminary Study", American Sociological Review, 28 (1), 1963, p. 55-67 ; Werner RAUB et Jeroen WEESIE, «Symbiotic Arrangements : A Sociological Perspective", Journal of Institutional and Theoretical Economics, 149, 1993, p. 716-724 ; Werner RAUB et Jeroen WEESIE (eds.), The Management of Durable Relations : Theoretical and Empirical Models for Households and Organizations, Amsterdam, Thela Publishers, 2000 ; Gerrit RoOKS, Werner RAUB, Robert SELTEN et Frits TAZELAAR, "How Inter-Firm Co-operation Depends on Social Embeddedness : A Vignette Study ", Acta Sociologica, 43, 2000, p. 123-137.

12. Emmanuel LAZEGA et Lise Mounier, «Interdependent Entrepreneurs and the Social Discipline of their Cooperation : Structural Economic Sociology for a Society of Organizations ", in Olivier FAVEREAU et Emmanuel LAZEGA (eds.), Conventions and Structures in Economic Organization: Markets, Networks and Hierarchies, Cheltenham (UK), Edward Elgar, 2002 ; Emmanuel LAZEGA, « Théorie de la coopération entre concurrents : Organisation, marché et analyse de réseaux ", in Philippe STEINER et François VATIN (dir.), Traité de sociologie économique, Paris, PUF, 2009 (à paraître).

13. Ian Ayres et John BratThWAITE, Responsive Regulation : Transcending the Deregulation Debate, op. cit. 
dépit des coûts élevés du règlement des litiges, les entreprises utilisent des mécanismes de règlement institutionnels formels 14 ; les conflits suivent la pyramide, transformant les plaintes informelles en procédures et décisions judiciaires ${ }^{15}$.

L'utilisation d'une approche organisationnelle de la polynormativité dans une institution de régulation conjointe est limitée. L'observation du travail des juges en général est difficile ; ils en parlent peu afin de protéger leur indépendance. De plus, la complexité des tâches accomplies par les juges du commerce, aussi bien dans le domaine du contentieux que dans celui des procédures collectives, renvoie aux compétences multiples (juridiques, économiques, gestionnaires, entre autres) mobilisées dans ce type d'institution consulaire. L'activité des juges est fortement définie et encadrée par la procédure, mais aussi marquée par les conflits de normes qu'ils ne cherchent pas nécessairement à exposer.

Pour mener une recherche empirique à caractère exploratoire, nous avons donc opté pour une approche jurisprudentielle à partir de cas d'espèce, visant à susciter de la part des juges l'explicitation de leurs critères de jugement. L'objectif était aussi d'observer la relation entre l'origine professionnelle des juges consulaires et leurs décisions de justice lorsque le droit en vigueur n'impose pas de solution et qu'ils doivent mobiliser leur pouvoir souverain d'appréciation ${ }^{16}$. Il a ainsi été possible de recenser quelques-unes des conventions sur lesquelles les juges s'appuient pour prendre des décisions de justice en matière économique.

Dans notre approche de la polynormativité, nous n'abordons pas la question des formes d'interaction entre le droit positif et les autres normes, question qui dépasse le cadre restreint et descriptif de cet article. Il ne s'agit pas ici de mettre au jour la place extrêmement variable du droit formalisé «étatique » dans le raisonnement des juges consulaires, mais de mettre au jour des conflits de normes en utilisant comme analyseur des situations où le droit laisse au juge consulaire un fort pouvoir d'appréciation.

14. Marc Galanter et Charles R. EPP, "A Beginner's Guide to the Litigation Maze», Business Economics, 27(4), 1992, p. 33-38 ; Terence DunworTH et Joel Rogers, "Corporations in Court : Big Business Litigation in U.S. Federal Courts 1971-1991 », Law \& Social Inquiry, 21 (3), 1996, p. 497-592 ; Ross E. CHEIT et Jacob E. GERSEN, «When Businesses Sue Each Other : An Empirical Study of State Court Litigation », Law \& Social Inquiry, 25 (3), 2000, p. 789-816.

15. William Felstiner, Richard ABel et Austin SARAT, «The Emergence and Transformation of Disputes : Naming, Blaming, Claiming », Law \& Society Review, 15 (3/4), 1980-1981, p. 631-654.

16. Nous ne procédons pas ici à une comparaison entre juges consulaires et magistrats de carrière. Notre propos n'est pas d'affirmer que les juges consulaires disposeraient, dans les textes, d'une marge d'action beaucoup plus considérable que celle d'autres juges. Voir à ce sujet les nombreux travaux sur le "pouvoir d'interprétation du juge ", par exemple : François OST, "Le rôle du juge. Vers de nouvelles loyautés ", dans Le rôle du juge dans la cité, Bruxelles, Bruylant, 2002, p. 15-45; Philippe GÉRARD, François OsT et Michel VAN DE Kerchove, Droit négocié, droit imposé ?, Bruxelles, Facultés universitaires Saint-Louis, 1996 ; François OsT, "L'herméneutique juridique entre hermétisme et dogmatisme. Le jeu de l'interprétation en droit", Revue internationale de sémiotique juridique, VI (18), 1993, p. 227-247. Il reste que les juges consulaires revendiquent pour la plupart la possibilité de s'appuyer pragmatiquement et explicitement sur leur propre expérience de la gestion, de l'économie et du monde des affaires pour prendre des décisions. Cette revendication et ce pragmatisme rapprochent souvent leurs décisions du jugement en "équité " plutôt que du jugement en droit. Voir à ce sujet Emmanuel LAZEGA, "Quatre siècles et demi de New Law \& Economics : du pragmatisme juridique dans le régime consulaire de contrôle social des marchés ", Revue française de socioéconomie, 3, 2009 (à paraître). 


\section{I.1. Des « usages » aux « sensibilités individuelles » dans la gouvernance du monde des affaires}

$\mathrm{Au}$ TCP, le problème de ces conventions est souvent réduit par les juges à celui des « usages » et des «normes » du monde des affaires. En effet, le droit des affaires ignore souvent les us et coutumes idiosyncrasiques désignés, dans les Tribunaux de commerce, comme des "usages » 17 basés sur les sous-cultures traditionnelles de l'industrie ou du commerce. Le Guide pratique du magistrat consulaire 18 définit un usage comme « une pratique qui, pour une profession et une région déterminées, a force obligatoire dans les contrats ». Il y a des usages locaux (par exemple, usages des ports de commerce pour la réception et la délivrance des marchandises expédiées par mer), des usages nationaux et des usages internationaux (par exemple, conditions générales de vente des fonderies européennes). Certains de ces usages ont été incorporés à la loi (par exemple, usages commerciaux sur les ventes commerciales), d'autres sont incorporés dans des contrats type (par exemple, les règles d'York et d'Anvers sur les avaries communes en transports maritimes). Si un usage commercial ne peut être contraire à une loi commerciale impérative, il peut par contre déroger aux règles du Code civil qui ne sont pas d'ordre public (par exemple, présomption de solidarité dans les opérations commerciales et capitalisation trimestrielle des intérêts en matière de comptes courants bancaires). S'il n'est pas de notoriété publique, l'usage doit être prouvé par celui qui l'invoque. Cette preuve peut prendre la forme d'une attestation délivrée par la chambre de commerce et d'industrie ou par un organisme professionnel faisant autorité comme l'Association française des banques 19 .

Les usages au sens défini par le Guide pratique du magistrat consulaire sont ainsi des normes sectorielles et des spécifications techniques reconnues et déposées au greffe du Tribunal. En particulier en matière de contrats, ces usages commerciaux guident aussi les opérations commerciales et sont reconnus formellement par le Tribunal de commerce. Bien que l'activité commerciale soit aujourd'hui fortement formalisée dans des contrats écrits et que le recours à ces usages et spécifications reste exceptionnel, les juges consulaires les mentionnent souvent pour souligner le rôle régulateur de leur institution.

Il reste que ces usages n'épuisent pas les normes, conventions et critères non juridiques auxquels les magistrats consulaires ont recours pour prendre des décisions de justice lorsqu'ils disposent d'un fort pouvoir d'appréciation. C'est notamment ce que montrent les débats au sein du Tribunal sur l'équilibre entre le jugement en droit et le jugement en équité. En effet, les juges consulaires insistent beaucoup sur la nécessité de prendre en compte, dans leur raisonnement pratique et dans le délibéré, l'expérience qu'ils ont du monde des affaires et de l'économie, expérience qui va de pair avec des jugements "en équité ». Le poids relatif du jugement en équité par rapport au jugement en droit semble varier d'un juge à l'autre. Pour les

17. On répète volontiers au Tribunal de commerce que ces usages sont « sources de droit ».

18. Guide pratique du magistrat consulaire, Tribunal de commerce de Paris, 2001, p. 134.

19. Cette attestation est dénommée "parère ». Depuis 1982, il existe au greffe du Tribunal de commerce de Paris (service des expertises) un bureau de dépôt des usages professionnels auprès duquel les professions ont été appelées à déposer leurs usages et règlements : il en existait 275 au 31 octobre 2000. 
uns, on doit évidemment apprendre à ne pas juger en équité. L'équité présuppose des raisonnements qui donnent prise à des influences extérieures affaiblissant l'indépendance du juge.

Le mauvais juge c'est celui qui n'a pas une formation juridique suffisante, donc il veut tout juger de bonne foi, en équité. L'équité, c'est le piège le plus terrible pour faire n'importe quoi. [...] Il ne faut pas que l'équité fasse faire n'importe quoi. Il y a un critère simple : il y a toujours un avocat qui envoie son dossier avant l'autre, donc vous vous dites qu'il a raison, et huit jours après c'est l'autre, et vous n'aviez pas du tout pensé comme lui. Donc vous vous référez au droit, puis quand vous avez statué en droit, tout bien pesé, vous essayez de le faire coïncider avec l'équité. Mais si l'équité passe avant le droit, vous ferez n'importe quoi et vous serez réformé par la cour d'appel derrière, vous aurez fait perdre du temps et de l'argent à tout le monde. (Juge 153.)

Pour les autres, c'est précisément la spécificité du Tribunal de commerce que de permettre de tenir compte de l'équité, même si elle pose des problèmes de définition. Beaucoup de magistrats consulaires tiennent parallèlement un discours différent qui met en avant leur pouvoir souverain d'appréciation et leur devoir moral de juger en s'appuyant sur leur connaissance non seulement du droit, mais aussi de l'économie, de la gestion et de leur expérience individuelle. Les entretiens avec les juges ont mis en évidence une conviction très ancrée chez eux concernant la nécessité de s'appuyer sur une synthèse de droit, d'une part, d'économie et de gestion, d'autre part.

Je suis juriste de formation, de profession, puisqu'en fait j'ai exercé tout le temps en entreprise, et il est évident que j'apporte au tribunal également la compétence de juriste, et lorsque vous avez des affaires qui sont des affaires un petit peu plus complexes en droit, je dis en droit parce que très vite on arrive à savoir, et peut-être beaucoup mieux d'ailleurs que les juges professionnels, on arrive à savoir ce qui s'est passé en réalité. Et ce n'est pas parce que vous avez la vérité que forcément la personne qui se plaint est dans son droit. Et c'est là où c'est très difficile, parce que la justice doit être rendue, et on rend justice en droit, alors on essaye de la rendre en droit et en équité, mais c'est pas toujours facile. Il est évident que c'est au Tribunal de commerce que vous avez le plus de chances d'avoir une justice rendue en droit et en équité. Et je crois que ça c'est un point qui tend à se perdre de plus en plus, et avec la réforme dont on parle, on s'éloigne de beaucoup des raisons pour lesquelles les Tribunaux de commerce ont été créés. (Juge 101.)

Un autre juge du TCP le formule encore plus nettement :

[...] Vous avez les tribunaux de l'ordre civil qui sont des professionnels du droit qui appliquent le droit stricto sensu. Nous, on doit voir le droit et les incidences économiques que ça a. Alors bien sûr, on ne peut pas avoir de jugements qui soient contraires totalement à la loi, mais on doit trouver un moyen terme... On est juge, mais juge du commerce, et on doit avoir une vue plus économique que purement juridique. (Juge 72 .)

Ainsi, les conventions ne se réduisent pas aux « usages " reconnus par le Tribunal. Dans le vocabulaire des magistrats, les « sensibilités individuelles " prennent le relais des usages recensés et du droit codifié pour adapter les décisions aux réalités toujours en mouvement de l'économie et des affaires. Le règlement efficace des conflits ne peut en effet passer outre tout un ensemble de conventions beaucoup 
plus informelles ${ }^{20}$ qui structurent de manière variable les pratiques du monde des affaires. On peut faire l'hypothèse qu'il est possible de retrouver, sous les sensibilités individuelles, des "sensibilités collectives ", c'est-à-dire des conventions partagées différentes des "usages" formellement recensés. Ces conventions sont très proches de ce que les sociologues appellent des représentations collectives.

\section{I.2. Des « sensibilités individuelles » aux représentations collectives}

La méthode d'enquête choisie pour accéder à ces conventions est basée sur le recueil des commentaires jurisprudentiels des juges consulaires au sujet de cas d'espèce réels délibérément choisis 21 dans des domaines où le droit ne donne pas de solution immédiate. Dans ces domaines, le juge doit mobiliser son pouvoir souverain d'appréciation, qui comprend potentiellement des "sensibilités " qui passent pour individuelles. Par ce moyen, on cherche à identifier la diversité des représentations collectives existant au sein du TCP, diversité qui renvoie à une lutte entre conventions sous-jacentes. Nous faisons en effet l'hypothèse que ces sensibilités caractérisent non pas simplement des individus isolés mais l'ensemble des individus provenant d'un secteur spécifique de l'économie. Parmi ces sensibilités, celles des juges provenant des secteurs de la banque et de la finance et ayant un diplôme en droit (banquiers-juristes) nous intéressent particulièrement parce qu'ils sont centraux dans le partage des compétences et de l'expérience caractérisant la communauté des juges du TCP pendant les années où nous avons pu l'approcher 22 .

\section{I.3. Méthodologie : trois cas d'espèce mobilisant le pouvoir souverain d'appréciation du juge}

Les juges doivent en effet rédiger leur jugement, c'est-à-dire (article 455 NCPC) exposer les faits, la procédure (les prétentions respectives des parties et leurs moyens en demande et en défense), les raisons (motifs) qui amènent la formation de jugement à statuer dans tel ou tel sens, la décision prise par le tribunal (énoncée sous forme de dispositif). Cette rédaction peut être complexe lorsque les jugements comportent plusieurs demandes ou concernent des domaines différents.

Prenant appui sur cette obligation de rédaction, les cas d'espèce présentés aux juges mobilisent leur pouvoir souverain d'appréciation, c'est-à-dire leur sensibilité, qui peut être personnelle, mais aussi culturelle et partagée par les membres de leur milieu socioprofessionnel, et qui doit donc s'appuyer sur des conventions ou représentations spécifiques à leur milieu d'origine. Au cours des entretiens, notre travail a été d'aider les magistrats à remonter vers ces conventions et à les rendre explici-

20. Emmanuel Lazega et Olivier FAVEREAU, « Introduction », in Olivier Favereau et Emmanuel Lazega (eds.), Conventions and Structures in Economic Organization: Markets, Networks, and Hierarchies, Cheltenham (UK), Northampton (Mass), Edward Elgar, 2002, p. 1-28.

21. En effet, nous n'avons pas eu accès aux données individuelles permettant de relier un juge à un jugement (données statistiques désagrégées, propriété du greffe du Tribunal, une entreprise privée).

22. Voir à ce sujet : Emmanuel LAZEGA et Lise Mounier (avec la collaboration de Ana Maria FALCONI), Partage des compétences entre magistrats consulaires et usages du monde des affaires au Tribunal de commerce de Paris, Rapport à la Mission de Recherche Droit et Justice, Paris, Ministère de la Justice, 2007 ; Emmanuel LAZEGa, Lise Mounier, Tom Snijders et Paola Tubaro, « Réseaux et controverses : de l'effet des normes sur la dynamique des structures », Revue française de sociologie, 49 (3), 2008, p. 467-498. 
tes. Les cas d'espèce de contentieux soumis aux juges consulaires sont des cas réels (jugés dans une autre juridiction commerciale) mais ne constituent pas des dossiers complets. Les cas ne sont présentés que sous la forme de jugements rédigés 23 et les juges, découvrant le jugement sur place, répondaient ensuite à nos questions ouvertes et fermées.

\section{L'enquête sur les juges consulaires du TCP}

Cet article s'appuie sur une étude du fonctionnement du Tribunal de commerce de Paris qui a été réalisée en trois temps et qui s'est centrée sur les juges consulaires. Une première étape, à l'automne 2000, à partir d'une liste de 157 juges présents au tableau des audiences auxquels 10 personnes - les «sages» et les responsables d'association ont été ajoutées. Pour la deuxième enquête, en 2002, une liste de 197 juges a été établie réunissant les juges consulaires contactés en 2000 ainsi que les magistrats élus à l'automne 2000 et 2001. 155 magistrats présents au tableau des audiences 2002 ont été contactés ainsi que 26 juges ayant quitté le Tribunal (fin de mandat après 14 ans ou démission). En 2005, nous avons procédé à une troisième étape ; une liste de 234 juges consulaires a réuni les présents au tableau 2005 (166) et les présents aux vagues précédentes ainsi que les élus depuis 2002. À chaque étape, nous avons tenté d'interviewer tous les juges. $94 \%$ d'entre eux ont répondu à nos questions en $2000,86 \%$ en 2002, et $91 \%$ en 2005. Les résultats présentés dans le présent article sont extraits de la troisième enquête (2005) consacrée au travail des juges ainsi qu'à la manière dont ils mobilisent pratiquement leur expérience du monde des affaires dans ce travail. Ces juges ont été interviewés par entretien semi-directif, de préférence sur leur lieu de travail (pour ceux qui sont encore en activité), à leur domicile ou dans leur bureau au tribunal, à leur convenance. Après avoir testé les cas d'espèce auprès de juges consulaires de la périphérie de Paris (Versailles, Bobigny, Nanterre), nous avons dû veiller à éviter deux écueils : premièrement, donner au juge le sentiment de tester son savoir juridique ; deuxièmement, mettre le juge en porte-à-faux par rapport au jugement de l'un de ses collègues. Nous avons rassemblé un volumineux corpus d'entretiens ainsi que des données statistiques. Le présent article rend compte des principaux résultats obtenus, articulant analyse qualitative et mesure quantitative 24 .

Les trois cas d'espèce portaient respectivement sur l'appréciation du préjudice et la concurrence entre les juridictions judiciaires et administratives, sur l'appréciation du trouble manifestement illicite ou du danger imminent, et enfin sur l'appréciation du report d'une assemblée générale extraordinaire en vue d'une expertise de gestion. Il s'agit de mener l'analyse du point de vue de la problématique de la relation entre conventions et structures dans le contrôle social polynormatif du monde des affaires. Dans la diversité des conventions mises au jour, un intérêt

23. Certains juges ont objecté qu'ils n'avaient pas assez d'informations pour commenter le jugement puisqu'un vrai dossier inclut les pièces, l'assignation, les plaidoiries. Nous leur avons répondu que les cas d'espèce ne servaient que de support pour les aider à exprimer leur philosophie et leur sensibilité personnelle sur ce qu'il faut faire dans des cas du même type, ceux où le droit ne donne pas de réponse immédiate. Notre propos n'est évidemment pas de remettre en question le principe de l'autorité de la chose jugée.

24. Pour davantage de détails sur la troisième étape de l'enquête et sur le guide d'entretien, voir Emmanuel LAZEGA et Lise MoUnIER (avec la collaboration de Ana Maria FALCONI), Partage des compétences entre magistrats consulaires et usages du monde des affaires au Tribunal de commerce de Paris, op. cit. 
particulier est porté aux positions prises par les magistrats banquiers-juristes qui constituent une bonne partie de l'élite « épistémique » du TCP 25.

Un caveat méthodologique s'impose avant la présentation des cas et des résultats. L'existence même de variations dans les réponses des magistrats ne nous étonnera pas puisque nous nous sommes délibérément placés dans des cas de figure mobilisant un pouvoir souverain d'appréciation et les sensibilités différentes qu'elles peuvent manifester. Ce qui nous intéresse d'abord, c'est la nature et l'origine de ces variations dans l'appréciation souveraine. Mais, répétons-le, l'exercice auquel les juges consulaires ont bien voulu se prêter n'est pas une simulation de jugement. Ils n'ont fait que lire et commenter un jugement, puis répondre à des questions générales, sans avoir accès au dossier. Or on connaît, en procédure commerciale, l'importance, par exemple, de l'assignation et la manière dont elle structure le dossier, notamment par la qualification des faits qu'elle propose.

Ces limites imposent à leur tour une certaine prudence dans l'interprétation des résultats. La principale difficulté d'interprétation tient au degré de précision des cas. Les cas que nous avons soumis aux juges sont relativement précis sur la problématique juridique, mais moins sur la situation de fait. La problématique juridique ne peut être ensuite appliquée à une situation de fait précise. Les résultats constituent donc une exploration touchant des questions de principe, exploration qui pourrait servir de base de départ pour des études ultérieures : soit une étude statistique des décisions du Tribunal dans ces domaines ; soit une étude approfondie présentant le même dossier complet devant trois magistrats chaque fois différents, des collégialités différentes, et observant l'analyse, l'appréciation des faits, la construction d'un consensus et la rédaction.

\section{De l'appréciation du préjudice en matière de concurrence déloyale}

L'un des domaines dans lesquels les juges consulaires disposent d'un large pouvoir d'appréciation est celui de l'appréciation du préjudice et l'évaluation des dommages et intérêts, notamment lorsque le préjudice est causé par des comportements de concurrence déloyale. L'évaluation du préjudice relève de l'appréciation souveraine du fond par le juge. Les juges évoquent la singularité de chaque cas et s'appuient sur le fait qu'elle n'est accessible qu'à eux seuls pour justifier leur libre pouvoir d'appréciation, notamment à l'égard des experts. Cette approche conduit donc nécessairement à des inégalités d'indemnisation. La réparation du dommage en droit des affaires est aussi complexe que celle des dommages corporels 26 . En

25. Nous considérons que le Tribunal constitue une sorte de "communauté épistémique " (ou plutôt le lieu d'intersection de plusieurs sous-communautés épistémiques) et que les juges les plus consultés par leurs pairs constituent une "élite épistémique " particulièrement influente dans cette institution. Voir à ce sujet : Emmanuel LAZEGA, The Micropolitics of Knowledge: Communication and Indirect Control in Workgroups, New York, Aldine de Gruyter, 1992 ; Emmanuel LAZEGA et Lise MOUNIER, «Interlocking Judges : On Joint External and Self-Governance of Markets", op. cit.; Emmanuel LAZEGA et Lise MouniER, "Quête de statut social, partage des compétences et néo-corporatisme au Tribunal de commerce de Paris », in Hélène MiChel et Laurent Willemez (dir.), La justice au risque des profanes, Paris, PUF, coll. « CURAPP », 2008.

26. On constate actuellement dans ce domaine un manque de cohérence qui n'a rien de surprenant étant donné toutes les incertitudes liées aux notions de réparation et de victime indemnisable, et de transparence des pratiques d'appréciation du préjudice, d'évaluation et de réparation. 
effet, dans le commerce comme ailleurs, se pose la question fondamentale du sens même de la réparation, d'autant que les acteurs de l'économie sont souvent des sociétés, c'est-à-dire des personnes morales (article 1832 du Code civil). Les dommages-intérêts ont-ils pour objet essentiel, voire unique, la « remise en état " dans toutes ses composantes (matérielles et morales, par exemple) ou visent-elles, dans le même temps, un effet de sanction répressive, cette dimension punitive étant habituellement réservée au droit pénal ?

\section{Cas d'espèce $\mathbf{n}^{\circ} 1$}

De l'appréciation du préjudice en matière de concurrence déloyale et du recours aux autorités administratives indépendantes

Une société anonyme au capital détenu entièrement par l'État (ci-après désignée «la Société $\mathrm{G} »$ ), active dans le secteur de l'armement et en particulier dans le secteur de la construction de chars de combat, a été assignée par une société concurrente (ci-après désignée «la Société $M$ ») au motif qu'elle aurait pratiqué des "prix prédateurs » sur le "marché des réducteurs de vitesse".

La Société $\mathrm{M}$ demandait à titre principal dans son assignation que le Tribunal condamne la Société G à lui payer la somme de 10762900 euros à titre de dommages et intérêts, et, à titre subsidiaire, la désignation d'un expert pour chiffrer le préjudice.

Le Tribunal a fait jouer son pouvoir souverain d'appréciation, et n'a pas nommé d'expert pour évaluer le préjudice.

Après un examen du taux de marge et de l'assiette du chiffre d'affaires retenus par la demanderesse, ainsi qu'une analyse de la perte de capacité concurrentielle et des préjudices moral et matériel, le Tribunal a retenu une évaluation du préjudice égale à moins de $3 \%$ de la somme initialement réclamée.

Sur le taux de marge, le Tribunal a ainsi considéré que «dans les industries lourdes, où la concurrence est sévère, les fabricants appliquent au coût de revient du matériel commandé une marge de 10 à $20 \%$ ", et a retenu un taux de $10 \%$.

Sur l'assiette du chiffre d'affaires retenu, le Tribunal a considéré que la Société $\mathrm{M}$ ne fournissait pas la preuve de ses allégations et a considérablement minimisé le préjudice afférent allégué.

Enfin, le Tribunal a constaté l'absence de tout préjudice moral et matériel, en considérant notamment que « les risques de litige sont inhérents aux affaires et peuvent survenir dans la vie d'une entreprise ".

Par un jugement avant dire droit, le Tribunal avait sollicité l'avis du Conseil de la concurrence afin d'obtenir des éléments objectifs pour statuer sur les demandes fondées sur les articles L 420-1, L 420-2 et L 420-5 du Code de commerce.

Le Tribunal s'est donc référé à cet avis dans son jugement : « [...] le Tribunal se satisfera de l'avis émis par le Conseil de la concurrence, et dira que la Société M ne rapporte pas la preuve d'une entente anticoncurrentielle de la part des parties défenderesses ni de l'abus de position dominante de la Société G et déboutera purement et simplement la Société M de son action fondée sur le droit de la concurrence ».

Les limites à la liberté de la concurrence sont inscrites dans les textes pénaux qui sanctionnent des pratiques déloyales comme la contrefaçon, la publicité mensongère, la tromperie sur la marchandise et la vente à perte. Sur le plan civil, sont notamment constitutifs de la concurrence déloyale la création d'une confusion entre une entreprise et sa concurrente (utilisation de signes distinctifs propres à cette entreprise, imitation de ses produits et créations), la tentative de désorganisation d'un concurrent (détournement de clientèle, débauchage abusif de personnel, 
utilisation frauduleuse de listes de clients ou de documents confidentiels), le dénigrement, les agissements parasitaires. La contrefaçon sanctionne une atteinte au droit de propriété, la concurrence déloyale un comportement particulier 27 . Pour approcher la diversité des sensibilités des magistrats consulaires dans ce domaine, nous avons utilisé le cas en question présenté ci-dessus.

Ce cas appelle une évaluation du préjudice matériel et une évaluation du préjudice moral ; il pose la question du recours à un expert et celle de l'opportunité d'un recours à l'avis d'une Autorité administrative indépendante (le Conseil de la concurrence). La décision du juge s'appuie notamment sur l'article 420-1 (Code de commerce) sur les pratiques anticoncurrentielles, et plus précisément le $\$ 32$ sur les prix prédateurs, i.e. «un prix de vente unitaire d'un produit inférieur au coût variable unitaire de celui-ci» 28 .

Nous avons constaté que les juges ne raisonnent pas tous de la même manière en matière d'évaluation de préjudice matériel et moral.

En ce qui concerne l'évaluation du préjudice matériel, un peu plus d'un tiers des magistrats consulaires (38 \%) aurait pris la même décision que le Tribunal - qui considère qu'il n'y a pas eu de préjudice matériel dans ce cas d'espèce. Une minorité $(15 \%)$ aurait pris une décision opposée. Une part importante de magistrats (38\%) déclare ne pas savoir ce qu'elle aurait fait, devant un cas similaire, étant donné l'absence du dossier complet. Parmi les juges qui se sont exprimés sur cette question du préjudice matériel (53\%), l'absence de dossier complet ne semble pas être la seule cause d'hésitation. La problématique des dommages et intérêts est présentée comme volatile :

Pour les dommages et intérêts, quand on écoute les demandeurs, c'est la tartine beurrée des deux côtés avec quatre couches. Pour avoir un million d'euros, il faut en demander dix. Entre le montant demandé et celui qui est accordé, vous pouvez avoir des distorsions de 1 à 20. Quand on demande des dommages et intérêts, il faut prouver qu'il y a eu préjudice, que le préjudice est en relation directe avec le litige et enfin justifier quantitativement le préjudice. Quelle est la réalité du grief? Sinon aucun dommage et intérêt. Et même quand ils sont justifiés, le Tribunal les estime différemment : pour le Tribunal, c'est la tartine sans beurre et sans confiture. (Juge 200.)

La question qui nous intéresse plus particulièrement ici, celle de la punitivité des magistrats consulaires, a pour indicateur l'évaluation du préjudice moral d'une personne morale. Il s'agissait de savoir si tous les juges du TCP reconnaissent dans leurs jugements, lorsqu'on le leur demande, le préjudice moral d'une personne morale. Procèdent-ils tous de la même manière à cette évaluation tant du préjudice que de son indemnisation? Ou au contraire, existe-t-il des conventions ou critères variables sur ces questions?

27. Nous n'aborderons pas ici la question des méthodes d'évaluation du préjudice et de leur éventuelle harmonisation, mais seulement celle de la diversité des conventions sur lesquelles s'appuient les juges dans leur raisonnement individuel précédant le délibéré collégial. Il faut noter que l'importance pratique du juge rapporteur et son influence dans la formation de jugement collégiale au TCP donne davantage de poids à ces conventions lors du pré-délibéré.

28. Voir aussi le cas des prix prédateurs constituant un abus anti-concurrentiel de position dominante (Art 420-2, §19 du Code de commerce, ainsi que Conseil de la concurrence nº 94-D-3D du 24 mai 1994). 
Les magistrats se sont beaucoup exprimés sur l'évaluation du préjudice moral. Une majorité de juges consulaires (62\%) se déclare favorable en général à la reconnaissance d'un préjudice moral d'une personne morale dans la mesure où le droit positif admet le préjudice moral des personnes morales et sa réparation à condition que l'existence du préjudice soit prouvée (article 1382). La majorité des juges met l'accent sur cette question, s'accordant pour dire qu'il appartient au demandeur d'établir sa demande :

Je suis favorable à la reconnaissance d'un préjudice moral si on me le prouve. Le problème, c'est que c'est difficile à prouver. On n'est pas au pénal. Un jour, quelqu'un m'a fait une turpitude épouvantable. Je ne l'ai pas attaqué parce que je ne saurais pas comment l'attaquer. Je n'aurais pas su chiffrer le préjudice. (Juge 202.)

À cet égard, nous avons recueilli un grand nombre de récits de « coups bas " reçus (et donc forcément donnés) dans la vie des affaires, récits précisément suscités par la question du préjudice moral.

On m'a raconté le cas d'un salaisonnier qui commandait des wagons entiers de porcs en Espagne. Un jour, il tombe sur un commerçant qui lui fait un coup tordu ; il lui a envoyé un télégramme : "Bloqué à la douane; marchandise non conforme, je mets mes frigos à votre disposition. » Il n'a pas discuté. Il a tout de suite répondu "Quel est votre prix? " Mais il n'a plus jamais fait affaire avec lui et il l'a dit autour de lui. Quelques années plus tard, il a eu l'occasion de lui rendre la monnaie de sa pièce. Il lui a envoyé le même télégramme «Bloqué à la douane ; marchandise non conforme, je mets mes frigos à votre disposition.» L'autre a répondu : «Bien joué, je ne l'ai pas volé. » (Juge 203.)

Ces récits de "coups bas " aboutissent plutôt, selon la plupart des juges qui se sont exprimés à ce sujet, à une morale du stoïcisme qu'à une morale de la vengeance : le préjudice moral fait partie de l'âpreté de la vie des affaires et on doit souvent accepter de renoncer à demander un dédommagement faute de preuve et d'évaluation du préjudice.

Dans les propos des magistrats, la reconnaissance en général du préjudice moral d'une personne morale prend appui sur trois définitions possibles. La première relève du pretium doloris ${ }^{29}$. Même si une personne morale n'a pas de sensibilité, certains juges considèrent que l'entrepreneur, en tant qu'individu représentant cette personne morale, peut être affecté au sens du pretium doloris. En principe, les juges tiennent donc à cette reconnaissance et à la possibilité d'imposer des dommages punitifs comme réparation d'une douleur, même si cette dernière est difficile à prouver. La deuxième possibilité renvoie davantage à l'atteinte portée à la réputation de l'entreprise, à la marque, à la notoriété, par exemple dans les cas de contrefaçon, atteinte difficilement chiffrable mais évoquant plus directement une perte de bénéfice ou de chiffre d'affaires.

29. Le pretium doloris désigne une cause de préjudice en réparation de laquelle la victime obtient une compensation financière pour les souffrances qu'elle a ressenties ensuite des blessures subies et ce, que ces souffrances aient été ou non la conséquence directe ou indirecte (interventions chirurgicales) d'un fait accidentel ou d'un mauvais traitement dont l'auteur doit répondre. Cette réparation se cumule avec l'indemnisation des autres chefs de préjudice tels que le préjudice physique, le préjudice moral ou le préjudice esthétique. Cf. Serge Braudo, Dictionnaire du droit privé français, disponible à l'adresse Internet http://www.dictionnaire-juridique.com 
Je ne peux pas vous donner de pourcentages, mais il y a deux écoles, à mon avis, toujours. Il y a ceux qui disent : "Le préjudice, il nous faut des éléments sérieux pour l'évaluer, sinon des clopinettes." Et d'autres qui disent: "Il faut sanctionner le contrefacteur ", et donc même si on n'a pas d'éléments, on sanctionne. On punit, on ne peut pas laisser faire, avec l'argument que si on met un euro d'amende ce n'est absolument pas dissuasif, pour les contrefacteurs, de faire de la contrefaçon, car finalement ça ne leur coûte pas cher. (Juge 143.)

Enfin, certains juges donnent au préjudice moral un troisième sens qui peut se résumer à une sorte de supplément du préjudice matériel. En d'autres termes, ces juges voient dans la reconnaissance du préjudice moral un élément compensatoire lorsque le préjudice matériel leur semble sous-évalué :

Ce qui peut se passer, c'est qu'on n'ait pas suffisamment d'éléments pour évaluer le préjudice matériel de façon claire, précise, et à ce moment-là on rajoute le qualificatif moral pour, je dirais, majorer la quantification qu'on fait du dommage... Parce qu'il nous semble qu'en équité, effectivement, le défendeur a été particulièrement inqualifiable, qu'il a utilisé des moyens tout à fait critiquables. Et à ce moment-là, on a la conviction que le préjudice de l'entreprise est supérieur, et qu'on a du mal à trouver des bases purement arithmétiques pour l'établir. (Juge 132.)

Une tendance nette se dégage : les juges qui ont le moins d'ancienneté dans le Tribunal ${ }^{30}$ sont davantage favorables à la reconnaissance du préjudice moral des personnes morales que les juges plus anciens. Ces "jeunes juges" fondent leur argumentation sur un lien entre le préjudice moral et le non-respect de la créativité et de l'innovation dans les entreprises, développant ainsi une conception de l'entrepreneur et de l'entreprise comme plongés dans un monde des affaires devenu anomique.

Le fait de reconnaître en général l'existence du préjudice moral des personnes morales ne signifie pas que les juges l'accordent en pratique. Concernant les affaires qu'ils sont amenés à juger, les chiffres s'inversent : $63 \%$ des juges refusent de reconnaître un préjudice moral dans le cas d'espèce qui leur a été soumis, se montrant donc en accord avec le jugement rendu. Seuls 11 \% des juges se sont déclarés favorables à la reconnaissance d'un préjudice moral dans le cas d'espèce $n^{\circ} 1$. Les juges acceptent donc le préjudice moral des personnes morales en droit et en théorie, mais beaucoup moins en fait et dans la pratique.

De l'avis de certains juges, il y aurait une réticence, profondément ancrée dans la culture consulaire, à accorder des dommages et intérêts pour un préjudice moral d'une personne morale. L'un des magistrats consulaires perçoit l'état d'esprit général de ses collègues de la manière suivante :

Il y a quand même une mentalité assez générale [au TCP] qui est : la morale d'un commerçant est dans son tiroir-caisse. Par conséquent, en ce qui concerne le préjudice purement moral, on est tous très réticents. Dans la pratique réelle, il y a peu de jugements où il y a préjudice moral. Mais ça, ça vient peut-être du fait qu'il y a peu de juges qui ont été dans des Chambres où on parle beaucoup de préjudice moral. Il n'y a pratiquement que la $15^{\mathrm{e}}$ Chambre, celle de la concurrence déloyale et contrefaçon, où il y a des préjudices où, de façon classique, on vient nous demander des préjudices

30. On parlera d'une tendance plutôt que d'une liaison significative. Rappelons que les juges ont passé en moyenne 6,2 années au Tribunal (1 à 13 années, écart type 3,3). 
moraux. Et cette chambre-là est quand même assez réticente au préjudice moral. Et ceux qui n’y ont pas été, ils n'ont pas été confrontés à ça. [...] Les autres sont dans des préjudices moraux, alors du type du cas d'espèce, et alors là quand même il ne faut pas se moquer du monde, euh... le préjudice moral, non. Alors que, en contrefaçon, c'est un peu plus sérieux, parce qu'il y a l'atteinte au droit patrimonial, euh... l'affection immense du créateur pour son modèle de jeans. Il y a les gens qui l'accordent, effectivement, de façon un peu sortie du chapeau. Souvent ils mettent : "Tous préjudices confondus ». Ce qui évite de trop détailler. (Juge 201.)

Plusieurs magistrats ayant siégé dans la Chambre spécialisée dans les affaires de concurrence déloyale et de contrefaçon notent en effet que peu d'affaires de ce type jugées dans cette chambre font l'objet d'une reconnaissance du préjudice moral pour une personne morale. Certains juges sont conscients du paradoxe et l'expliquent par le clivage entre les collègues qui ont une tendance à s'attribuer un rôle de justicier et ceux qui suivent les règles du droit. Ce rôle de justicier est parfois évoqué de manière plus générale, comme relevant de la sphère du politique :

Les juges plus récents sont plus proches du monde de leur activité professionnelle, et disent : c'est vrai, on a quand même eu des préjudices moraux. [...] On peut penser qu'il y a même beaucoup de juges, y compris d'ailleurs chez les magistrats de carrière, qui considèrent qu'ils ont été mis sur terre pour y rétablir la justice. Ceux-là punissent. Et puis il y a ceux qui s'accrochent désespérément aux règles de droit et qui disent : il faut un préjudice, une faute, il faut un lien entre les deux, donc chiffrez-moi le préjudice. Et quand même, sans être trop méchant vis-à-vis de ceux qui sont venus sur terre pour rétablir la justice, il y a une grosse tendance au niveau de la Cour de cassation de combler des lacunes du droit politique, de la législation, en accordant, en entérinant des dommages et intérêts qui ont un caractère politique, en fait. Donc il y a quand même toute une tendance jurisprudentielle à accorder des dommages et intérêts qui en fait sont politiques, même s'ils ne le disent pas. [...] Ça n'est pas récent, ça date. Mais c'est continu, il n'y a pas de retournement de jurisprudence. Par exemple, la Cour de cassation a admis que le seul fait de faire de la contrefaçon induit un préjudice. Donc ils entérinent les arrêts de Cour d'appel qui disent : il y a contrefaçon, donc préjudice qu'on évalue à un chiffre pas toujours fondé. Il y a toute une tendance jurisprudentielle à donner des pouvoirs punitifs au juge. Beaucoup d'entre nous se situent quand même dans cette tendance. Mais, à la limite, je crois que le tribunal de commerce en fait résiste plus à cette tendance que les magistrats de carrière. Les magistrats de carrière observent plus attentivement que nous les arrêts de Cour de cassation parce qu'ils veulent leur avancement, je suppose. (Juge 203.)

D’autres disent accorder des dommages et intérêts pour préjudice moral précisément pour compenser l'impossibilité avérée de prouver des préjudices matériels. La concurrence déloyale, par exemple, est tellement difficile à prouver que certains juges accordent un euro symbolique, alors que d'autres, pour les mêmes raisons, accordent des millions d'euros, ce qui revient le plus souvent à considérer les dommages et intérêts comme punitifs ${ }^{31}$.

31. Voir par exemple http://lexinter.net/ACTUALITE/lvmh_morgan_stanley.htm pour l'affaire LVMH vs. Morgan Stanley (12 janvier 2004). 
En résumé, l'approche « restitutive » du préjudice et l'approche « répressive » 32 ou " punitive » sont toutes deux présentes au TCP. L'approche restitutive est populaire au commerce parce qu'elle convient bien à l'idéologie du rétablissement d'un lien entre l'auteur de l'infraction et sa victime. Les juges consulaires déclarent se sentir proches des entrepreneurs, tous appartenant à un même monde des affaires qui a ses règles, son rythme, ses pratiques. Mais l'approche punitive a aussi du succès. L'idée principale est que le préjudice individuel subi dans le cas d'espèce $\mathrm{n}^{\circ} 1$ s'accompagne d'un préjudice collectif parce qu'il implique la destruction des circuits «naturels » du marché. La question se poserait donc en termes de responsabilisation des commerçants. Plusieurs juges en concluent que si la faute et la mesure de sa gravité ne sont pas présentes, il y a un risque fort de déresponsabilisation des pratiques commerciales.

\section{De la prolongation du contrat contre la volonté d'une des parties}

Un deuxième domaine où sont visibles des conflits de conventions et de représentations est celui de l'interventionnisme des juges dans le fonctionnement des marchés. Le droit des affaires repose sur une théorie générale des obligations. Les liens juridiques existant entre les parties peuvent découler d'obligations, soit contractuelles, soit extra-contractuelles. En matière commerciale, le contrat se forme par accord des parties sur ses éléments essentiels. Il n'est pas nécessaire qu'il y ait un acte écrit, sauf dans certains cas où la loi l'impose (par exemple, cautionnement, transaction, assurance, cession de fonds de commerce, etc.). L'article 1108 du Code civil énumère les conditions essentielles pour la validité du contrat: le consentement de la personne qui s'oblige, sa capacité de contracter, un objet certain, une cause licite dans l'obligation. Ainsi, le juge peut être amené à évaluer la capacité de certaines personnes à contracter, le caractère déterminé, possible et licite de l'objet de l'obligation. Entre commerçants, la preuve du consentement est libre et peut être établie par tous moyens. En cas de litige, le juge ne doit pas s'arrêter au sens littéral des termes de la convention, mais doit rechercher quelle a été la commune intention des parties (article 1156 du Code civil). Le juge doit interpréter les clauses ambiguës, douteuses ou non exprimées, en respectant un certain nombre de règles (articles 1157 à 1164 du Code civil) : par exemple, il doit suppléer les clauses d'usage, même si elles ne sont pas exprimées; dans le doute, une convention doit s'interpréter en faveur du débiteur de l'obligation dont l'exécution est demandée. Le juge est amené, par exemple, à se prononcer sur l'exécution des contrats (exécution forcée ; nullité, résolution, résiliation, caducité ; suspension de l'exécution; responsabilité et dommages-intérêts en cas de non-exécution ou de mauvaise exécution créant un préjudice à l'une des parties ; fixation d'intérêts moratoires ; application de clause pénale ou modification du montant de cette clause ; octroi de délais), sur la solidarité qui doit exister entre plusieurs personnes répondant d'une même obligation envers un même contractant, sur les obligations extra-

32. Pour reprendre à nouveau les concepts de «sanction répressive » et « sanction restitutive » que Durkheim, entre autres, explicite dans De la division du travail social (fin du chapitre I), et de droit répressif/restitutif (dans le chapitre VII). 
contractuelles (délits et quasi-délits, abus de droit, quasi-contrats (article $1372 \mathrm{du}$ Code civil), sur l'extinction des obligations et des créances (paiement-subrogation, compensation, novation).

Dans ce domaine, les juges du commerce disposent d'un pouvoir d'appréciation du trouble illicite ou du dommage imminent et, le cas échéant, du pouvoir de le prévenir ou de le faire cesser en ordonnant l'exécution d'une obligation, même s'il s'agit d'une obligation de faire (article 873 du Nouveau Code de procédure civile). Dans le cas présenté ci-dessous, il s'agit d'une mesure conservatoire prolongeant une garantie financière devant le péril imminent que provoque la brusque cessation, par un fonds de garantie, d'une caution qu'il accordait depuis plusieurs mois aux demandeurs. Nous avons considéré que la décision prise par le juge et la manière dont il/elle utilise son pouvoir d'appréciation dans ce domaine reflètent nécessairement un degré plus ou moins fort d'interventionnisme dans les marchés - interventionnisme que les juges s'interdisent en théorie.

\section{Cas d'espèce $\mathbf{n}^{\circ} 2$}

De l'appréciation du trouble manifestement illicite ou du dommage imminent dans un cas de prolongation du contrat contre la volonté d'une des parties

Plusieurs établissements du secteur de la scierie (ci-après désignés " les Demandeurs ») ont assigné en référé d'heure à heure un fonds de garantie (ci-après désigné «le Fonds ») avec lequel un accord-cadre avait été signé, permettant notamment aux Demandeurs d'obtenir les cautions nécessaires à l'obtention des marchés de coupes de bois de l'ONF. Devenu déficitaire, le Fonds en avait informé les Demandeurs fin mai 1997, mais avait continué fin juin à encourager les industriels, par voie de presse, à « introduire leurs demandes de caution pour les ventes d'automne ». Le Fonds avait finalement indiqué qu'il n'accorderait plus ses cautions après le 29 août, refus à l'origine de l'action des Demandeurs.

Les Demandeurs, à l'appui de leur procédure de référé, ont invoqué la parole donnée, la brièveté du délai de prévenance, alors qu'un délai de deux à trois mois aurait été raisonnable et constitue un délai de rupture fréquemment admis. Ils ont donc réclamé que le Fonds produise les cautions relatives à chaque lot obtenu.

Le Fonds a invoqué en revanche le fait qu'il avait prévenu les Demandeurs suffisamment tôt pour que ces derniers aient recours à d'autres solutions. En outre, le Fonds considérait qu'il ne représentait qu'une faible partie des cautions obtenues d'autres garants, et contestait dès lors l'existence d'un péril imminent au sens de l'article $873 \mathrm{du}$ nouveau Code de procédure civile.

Le Président du Tribunal a retenu cependant que :

- le Fonds avait invité la profession à maintenir ses demandes de caution, et avait envoyé aux Demandeurs la lettre de préavis de rupture très peu de temps avant le début de la campagne de coupes de bois ;

- la durée indéterminée des promesses de caution formées par le Fonds ne le dispensait pas, pour mettre fin au contrat, de respecter le délai de prévenance d'usage dans la profession ;

- le fait que le Fonds devienne déficitaire ne constituait pas une cause de rupture de l'accord-cadre.

En conséquence, le Président du Tribunal a ordonné, sur le fondement de l'article 873 alinéa 2 du Nouveau Code de procédure civile, sous astreinte, que le Fonds honore jusqu'au $1^{\text {er }}$ novembre 1997 les obligations de donner les cautionnements qu'il s'était engagé à fournir aux Demandeurs. 
Ce type de situation pousse souvent les juges à se positionner en tant que médiateurs, l'idée étant que les différends entre entreprises sont des conflits devant se régler plutôt par le dialogue ou la conciliation que par une décision juridictionnelle.

Non, moi en principe, moi je pense que les obligations de faire, tout simplement, se résolvent en dommages et intérêts, donc je serais plus enclin à octroyer au demandeur des dommages et intérêts, plutôt que d'obliger le Fonds à honorer, à poursuivre un contrat qui $a$ priori... voilà, c'est comme ça que je vois les choses. Bon, alors ça c'est le droit, mais il faut voir aussi si économiquement ce n'est pas absurde, je pense qu'il peut y avoir un décalage entre ce qu'il faut faire en droit et la bonne solution économique. On arrive sur des terrains difficiles. Alors, dans ce cas-là, moi j'adopte toujours la même solution, c'est-à-dire que je dépense une énergie considérable pour concilier les parties. Parce que, quand je pense que la solution en droit, et cela ça arrive finalement assez souvent, est mauvaise pour les deux parties, je les invite à se mettre d'accord. Et ça marche assez souvent, enfin sauf si une des parties est bornée. Dans le cas d'espèce, c'est un bon exemple, le préjudice subi par les demandeurs peut être considérable, c'est-à-dire que c'est un effet de dominos, donc condamner le défendeur à couvrir ce préjudice peut être démesuré pour lui par rapport à l'effort qu'il a à faire pour qu'il n'y ait pas de préjudice. Donc, moi, je cherche à concilier.(Juge 70.)

Les magistrats sont ici - par rapport au cas d'espèce précédent - beaucoup moins partagés dans leurs réponses. Les trois quarts d'entre eux (114/151) auraient pris la même décision que le Tribunal : ils auraient désigné un expert, considéré que certains faits reprochés par les Demandeurs au Fonds de garantie étaient avérés, et maintenu le contrat en vie par une injonction de faire. Leurs justifications sont de trois types.

La première s'appuie sur l'idée d'un respect nécessaire de règles élémentaires dans le monde des affaires :

Quand on a donné sa parole, effectivement, même s'il n'y a pas eu d'écrit comme dans le cas présent, il fallait maintenir le même délai. C'est important que ce qui avait été promis soit tenu et donc le fait que le Fonds honore jusqu'au premier novembre, par rapport au 29 août, on a septembre, octobre et novembre, ça me paraît assez normal. [...] On n'arrête pas, c'est trop facile ! C'est trop facile d'arrêter comme ça, d'un seul coup ! Un fonds de garantie comme ça ! C'est trop facile! Il faut être respectueux des entreprises, des entrepreneurs, et là, ils n'ont pas été respectueux. Et c'est trop facile de dire : j'arrête du jour au lendemain! (Juge 85.)

La deuxième est celle du respect des usages et habitudes professionnelles propres à chaque secteur économique :

Il faut savoir s'ils ont été pris au dépourvu, dans leurs habitudes professionnelles. $\mathrm{Si}$, par exemple, c'était le 10 juillet, et que, en trois semaines, ils peuvent retrouver des cautions, ça veut dire qu'entre le 10 juillet et le $1^{\text {er }}$ août, ils avaient tout le temps de trouver quelqu'un d'autre. On ne peut pas juger uniquement selon les règles de droit, il faut aussi juger selon les habitudes professionnelles, et donc savoir si les scieurs ont été pris complètement à contre-pied, ou si, au contraire, ils avaient réellement le temps de se retourner, et ça, ça ne peut être qu'en fonction des habitudes professionnelles. Donc a priori je pense que cette décision est une bonne décision, sous réserve de bien vérifier à quelle date exacte le Fonds a prévu que c'était fini, à savoir ce qui a été écrit fin mai, si c'était une intention, ou si c'était seulement: "Nous allons le faire ", et si le communiqué de presse de fin juin était un communiqué de presse très général, qui n'annulait pas ce qui avait été dit fin mai, et si les scieurs ont vraiment pu être abusés parce que, là aussi, les vrais professionnels savent exactement ce qu'il faut 
lire dans les publicités; donc tout ceci demande une analyse professionnelle, du jugement, et là-dessus, un juge a priori il n'y en a pas un seul qui dans tout le Tribunal connaît les problèmes de Scierie, mais il faut essayer de trouver un professionnel et contrôler auprès de lui que c'est bien ça. (Juge 104.)

De leur côté, les juges qui expriment leur désaccord avec la décision du Tribunal s'appuient sur deux types d'arguments. Pour les uns, le jugement du Tribunal relève de l'équité plus que du droit, ce qui serait suffisant pour le désapprouver.

Cette décision, c'est un peu contraire au droit. C'est de l'équité. Pour défendre les petits, la veuve et l'orphelin. Parce que c'est ça. Il y a une tendance générale à dire et faire la charité pour les petits. J'ai beau leur dire que ça fait trois mille ans qu'on leur a dit ce qu'il ne fallait pas faire, je n'ai aucun succès. C'est dans les décisions et dans l'esprit. Et ne croyez pas que les juges de carrière ou les autres appliquent le droit. Non. (Juge 201.)

Pour les autres, c'est au nom du pragmatisme des tribunaux consulaires, c'està-dire de la conscience des conséquences de la décision sur l'activité économique des entreprises, qu'il aurait fallu parvenir à une décision différente de celle du Tribunal.

L'un demande la résiliation, l'autre s'y oppose? Au plan de la réalité, un contrat ça ne peut se réaliser que si on est deux au départ et deux à l'arrivée. C'est comme le mariage. Il faut être réaliste : on ne peut pas poursuivre un contrat avec quelqu'un qui ne veut pas le poursuivre. Obliger quelqu'un à poursuivre un contrat contre son gré, c'est de mauvais augure. Il y aura des représailles, de la mauvaise foi, etc. On ne peut pas faire boire un âne qui n'a pas soif. Il va le faire dans un esprit préjudiciable à la poursuite du contrat. Le vrai problème de la résiliation, c'est que le Tribunal constate la résiliation, mais « aux torts de qui ?» Cela exige une analyse. Le Tribunal constate que le contrat est résilié aux torts et griefs de M. Tapdur ? Les griefs sont parfois partagés. Le vrai problème de la résiliation n'est pas d'obliger à poursuivre, mais de dire qui est responsable de la résiliation et quelles sont ses conséquences financières. [...] $\mathrm{Si}$ j'avais été scieur et que j'apprenais que ce Fonds est déficitaire, je chercherais un autre cautionneur. Faire une demande de caution, ce n'est pas l'obtenir automatiquement. Le Fonds n'est pas tenu d'émettre une caution en faveur de n'importe quel scieur. Le Fonds n'est pas tenu de distribuer toutes les cautions qu'on lui demande. Ça n'est pas automatique, donc ça me gêne là. Les demandeurs ont une position faible. Toutes les banques délivrent des cautions. Ou alors on les a prévenus tardivement et ils n'ont pas eu le temps de se retourner. [...] Ceci relève davantage des dommages et intérêts parce qu'il y a une perte de chances d'obtention de marchés. J'aurais eu tendance à considérer qu'en fait, le Fonds, s'il a donné un préavis infiniment trop court par rapport aux usages, les scieurs ont perdu une chance, ceci constitue pour eux un préjudice matériel qui ne peut s'indemniser que sous la forme de dommages et intérêts. (Juge 200.)

Les conventions mobilisées par les juges reflètent donc deux positions différentes. Celle qui prolonge le contrat contre la volonté des parties indique une disposition forte à l'interventionnisme dans les marchés. Celle qui laisse le contentieux se résoudre éventuellement en dommages et intérêts indique plutôt un interventionnisme faible dans les marchés. 


\section{Des litiges entre actionnaires et de l'intérêt social de la société commerciale}

Les conflits de conventions et de représentations au TCP sont repérables dans un troisième domaine, à savoir celui de l'interventionnisme des juges dans les sociétés, en particulier dans les conseils d'administration. Les litiges entre sociétés commerciales (qui sont régies par les articles 1832 à 1844-17 du Code civil et par les articles L 210-1 à L 252-13 du Code de commerce) sont de la compétence spécifique du Tribunal de commerce 33 . Mais la vie de chaque société commerciale (fonctionnement de ses organes, comme le conseil d'administration; dissolution) donne aussi lieu à d'autres contentieux. Ces sociétés commerciales peuvent prendre des formes multiples (S.N.C., S.A., S.A.R.L., S.A.S., société en participation, société créée de fait, G.I.E., G.E.I.E., etc.). La responsabilité des associés et des dirigeants, les fautes qu'ils commettent et dont ils sont personnellement responsables entraînent souvent leur mise en cause et des actions en justice - devant le tribunal de commerce lorsqu'il est question de leur responsabilité civile. La transmission ou la cession de parts et d'actions revêt aussi un caractère commercial si elle entraîne la cession du contrôle de la société ; le contentieux de cette cession relève de la compétence du tribunal de commerce et d'un régime juridique élaboré.

Dans ce contexte, le droit des sociétés cherche à privilégier le collectif (l'« intérêt social » de l'entreprise) sur l'individuel. L'intérêt social est l'intérêt de l'entreprise organisée comme personne morale avec une autonomie juridique poursuivant ses fins propres, dans l'intérêt commun des actionnaires (majoritaires ou minoritaires), des salariés, des créanciers et autres personnes intéressées pour en assurer la prospérité et la continuité 34 . Du fait des incertitudes entourant cette notion, les juges du commerce disposent d'une grande marge d'appréciation quand il s'agit de définir l'intérêt social d'une société 35 .

L'un des principes du droit des sociétés est donc qu'il existe un intérêt commun et que cet intérêt doit être respecté par tous les actionnaires. Ce principe crée un vrai débat entre les actionnaires d'une société, en particulier lorsque l'actionnariat est composé à la fois de petits groupements de personnes et de grosses sociétés cotées en bourse. Le juge doit privilégier l'intérêt social si la survie de la société est en cause. Si le litige risque de compromettre la vie de la société, le juge doit identifier et prendre en compte l'intérêt social. Le cas de conflit entre dirigeants, ou

33. Le Guide rappelle que ces textes ont été modifiés à de multiples reprises par un nombre croissant de lois et décrets. Ce mouvement législatif et réglementaire s'est considérablement accéléré au cours des dernières années, d'une part pour mettre le droit français en conformité avec les directives communautaires européennes, d'autre part et surtout par la volonté du législateur, face à la montée considérable de l'épargne publique en titres de sociétés, d'assurer une plus large information et une meilleure protection des sociétaires, en particulier des minoritaires, mais aussi des tiers.

34. Voir à ce sujet Dominique SCHMIDT, « De l'intérêt social », JCP E, 1995 I, 488. Voir, par exemple, au sujet de cette notion très générale et du débat sur la question de savoir si une société est plus une institution qu'un contrat (du fait de l'existence d'une intervention minutieuse du législateur dans les règles de son fonctionnement) : Jérôme Bonnard, Droit des sociétés, Paris, Hachette, $3^{\text {e }}$ éd., 2005 ; Alexis Constantin, Droit des sociétés, Paris, Dalloz, coll. " Mémentos Dalloz », 2005 ; Maurice CozIAN, Alain Viandier et Florence

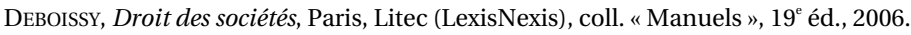

35. Sur la distinction entre intérêt social et intérêt commun, voir Dominique SCHMIDT, Les conflits d'intérêts dans la société anonyme, Paris, Joly éditions, coll. «Pratique des affaires », 2 e éd., 2004. 
même entre actionnaires, qui empêche le bon fonctionnement de la société et qui la met en péril, peut être un cas de nomination par le juge d'un administrateur provisoire (a priori indépendant des majoritaires et des groupes de pression). Un autre principe est que le juge ne doit pas s'immiscer dans la gestion de la société ou donner a priori raison ou tort à l'une des parties.

\section{Cas d'espèce $n^{\circ} 3$ \\ De l'appréciation du report d'une assemblée générale extraordinaire en vue d'une expertise de gestion dans un cas de litiges entre actionnaires}

Les actionnaires minoritaires (ci-après désignés « les Demandeurs «) d'une société (ciaprès désignée «la Société «) qui envisage d'effectuer un «coup d'accordéon » afin de reconstituer ses capitaux propres devenus inférieurs à la moitié du capital social, ont assigné cette dernière en référé d'heure à heure.

Les Demandeurs ont sollicité du Président du Tribunal statuant en la forme des référés, d'une part, qu'il ordonne le report sine die de l'assemblée générale extraordinaire jusqu'à ce qu'un rapport d'expertise soit déposé, et, d'autre part, qu'il désigne un expert chargé d'analyser la situation financière de la Société, dont en particulier :

- l'état de ses pertes ;

- la valeur de ses titres ;

— la valeur de la participation des Demandeurs dans la Société.

Les Demandeurs souhaitaient également que l'expert nommé par le Président du Tribunal donne son avis sur l'opportunité des opérations visées dans le rapport du conseil d'administration et sur le séquencement proposé par ses dirigeants pour ces opérations.

Le Président du Tribunal a constaté que les conditions étaient requises pour voir désigné un expert de gestion car :

- le Président de la Société était dans la cause et avait conclu en sa qualité de dirigeant de Société;

— des éléments satisfaisants n'avaient pas été communiqués en réponse aux questions posées à la Société par les Demandeurs.

Par ailleurs, le Tribunal a constaté que la désignation d'un expert s'inscrivait dans l'intérêt de l'ensemble des actionnaires, qu'ils appartiennent, ou non, à la majorité. De plus, le Tribunal a considéré que certains faits reprochés par les Demandeurs à la Société étaient avérés, et qu'en particulier :

— le pacte d'actionnaire semblait ne pas avoir été respecté ;

- les modalités des opérations en capital, et notamment le fait qu'une incorporation de réserves intervienne postérieurement à la réduction du capital assortie d'une augmentation du capital en espèces, semblaient ne pas avoir été clairement justifiées.

Relevant l'ensemble de ces éléments, le Président du Tribunal a donc décidé d'ordonner le report de l'assemblée générale extraordinaire et désigné un expert afin qu'il effectue une expertise de gestion.

Le cas du "coup d'accordéon" utilisé ici mobilise à nouveau les dispositions particulières du Nouveau Code de procédure civile (article 873, alinéa $1, \mathrm{n}^{\circ} 10$ ), à savoir le pouvoir du juge à faire cesser un trouble manifestement illicite. Dans ce cas d'espèce $\mathrm{n}^{\circ} 3$, le Tribunal est saisi d'une demande de report d'une assemblée générale extraordinaire et de nomination d'un expert de gestion pour vérifier le bien-fondé d'une opération de refinancement d'une entreprise décidée par son 
conseil d'administration ${ }^{36}$. Nous avons considéré que la décision prise par le juge et la manière dont il/elle utilise son pouvoir d'appréciation dans ce domaine reflétait un interventionnisme plus ou moins fort dans les conseils d'administration.

Ce type de cas se présente au TC tous les ans, notamment à l'approche du 30 juin, date à laquelle les entreprises doivent avoir tenu leur assemblée générale. Les juges utilisent toute la latitude dont ils disposent pour définir l'intérêt social de l'entreprise. Ils identifient pour cela différentes difficultés soulevées par la définition de l'intérêt social et par la prétention des parties à parler en son nom. D'une part, les décisions de l'assemblée expriment la volonté de la personne morale; le fait de la retarder risque de mettre la société en difficulté, c'est-à-dire en cessation des paiements. C'est alors la stratégie de la direction qu'il s'agit de clarifier :

Les affaires obéissent à une science mathématique: c'est la théorie des jeux. Quand un président de société est attaqué comme ça, il sait qu'il va l'être. Ou alors c'est un mauvais président. Il tente le coup. Le juge sait qu'il sait. Le juge peut juger que la demande des minoritaires est abusive, mais sinon le juge se dit : « Je sais que tu sais, mon gars. Tu as pris ton risque.» C'est lui qui a mal géré, ce n'est pas une intervention de la justice. Le gars a fait un faux pas volontairement et un expert va en décider. (Juge 202.)

Mais, d'autre part, des actionnaires minoritaires peuvent se demander s'ils vont devoir "mettre la main au porte-monnaie", même s'ils ne sont pas dans l'obligation de participer à l'augmentation du capital. Par exemple, l'un des juges traduit dans ses propos l'inquiétude des actionnaires minoritaires vis-à-vis des actionnaires majoritaires :

La question est de savoir si les actionnaires majoritaires ne font pas ce coup d'accordéon pour faire rentrer de nouveaux capitalistes, s'il s'agit en réalité d'une cession déguisée. La prétendue augmentation du capital serait faite pour vider les minoritaires et obtenir un contrôle sans eux; dans ce cas, l'opération qui n'aurait pour but que d'exclure des minoritaires serait une opération abusive. Si c'était une incorporation, pas l'entrée d'un tiers, le cas est quand même limité à une hypothèse plus sereine.

Ainsi, le conflit entre minoritaires et direction peut être dû aux inquiétudes légitimes des minoritaires en cas de mauvaise gestion, à la préparation d'une cession déguisée. Il pourrait aussi être dû à une petite vengeance des «minoritaires opprimés »:

Les petits actionnaires sont-ils brimés par un président omnipotent? Les demandeurs sont des "minoritaires opprimés ». C'est un cas pour Monsieur Neuville, le défenseur des "minoritaires opprimés"! Heureusement que c'est rarissime que n'importe quel petit actionnaire demande un expert parce qu'il ne sait pas ce qui s'est passé dans la société ! Sous réserve de tout ça, la demande me paraît excessive et même un peu bloquante. De toute façon, il faut faire une AGE. Le coup d'accordéon, c'est fréquent. C'est le commissaire aux comptes qui tire la sonnette d'alarme. Peutêtre que les petits actionnaires ne voulaient pas suivre l'augmentation du capital et qu'ils ont décidé de faire la guerre aux gros dirigeants. Mais, dans ce cas-là, on vend ses actions! C'est le genre d'initiative qu'il ne faut sûrement pas multiplier ! À tout

36. Les magistrats consulaires sont familiers de ce type de problème : «L'intervention d'un juge des référés dans le fonctionnement quotidien des sociétés commerciales doit rester exceptionnelle afin de ne pas porter atteinte aux règles voulues par la volonté des parties dans les statuts » (TCP, 18 février 1993 : JCP 93, $1,3682, n^{\circ} 2$, obs. Viandier et Caussain). 
moment, n'importe qui peut bloquer le fonctionnement de toutes les sociétés. En cas de malversation ou de fraude, je comprendrais. Ici, est-ce que c'est vraiment fondé ou est-ce une petite vengeance à l'égard d'un dirigeant qu'on ne peut pas sentir, pour finalement déboucher où, sur quoi ? (Juge 200.)

Un autre juge prend la position inverse :

Le Tribunal statue en droit. Si c'est le petit qui est bien fondé, c'est lui qui aura une solution favorable. La survie n'est pas un but en soi aux dépens des actionnaires car, à la limite, ce serait faire passer les salariés avant les propriétaires, comme en URSS, où c'était l'État. On statue en droit pour dire celui qui a tort. S'ils viennent au Tribunal, c'est qu'ils considèrent déjà que leur conflit met en jeu la survie de leur société. (Juge 204.)

Les conventions sur lesquelles les juges s'appuient pour raisonner sur ce cas d'espèce reflètent donc des positions différentes : celle qui cherche à éviter d'interférer avec la direction et celle qui témoigne d'une disposition forte à l'intervention dans le conseil d'administration.

\section{Polynormativité et conflits néo-corporatistes}

En résumé, cette approche du travail des juges consulaires cherche à vérifier l'hypothèse d'une relation entre leur carrière, leur appartenance sectorielle et la sensibilité qu'ils mobilisent dans des affaires où ils disposent d'un pouvoir souverain d'appréciation. Les cas d'espèce n'étaient donc utilisés que comme supports d'une réflexion plus générale sur les effets de la polynormativité contemporaine. Ils ont été sélectionnés et utilisés pour répondre à la question suivante : les sensibilités différentes des juges - celles qui s'expriment lorsque les magistrats mobilisent leur pouvoir souverain d'appréciation - sont-elles purement individuelles ou témoignent-elles de la présence, dans l'esprit des juges du TCP, de représentations et de dispositions plus collectives? Les comptages statistiques les plus élémentaires présentés dans le tableau 1 permettent de répondre à cette question pour chaque cas d'espèce séparément.

En ce qui concerne le degré de punitivité des juges, l'analyse des profils de leurs réponses met au jour quelques régularités. Notons, par exemple, que les juges qui ont le plus d'ancienneté au sein du Tribunal ont tendance à ne pas suivre le jugement en matière de préjudice matériel ; ils ont tendance à être moins punitifs que les juges les plus récents. En revanche, les banquiers-juristes ont tendance à le suivre et à minimiser le préjudice matériel (se ralliant à la décision présentée dans le cas d'espèce). De plus, dans ce cas précis, la totalité des magistrats ayant fait du droit et provenant du secteur de la banque et de la finance considèrent qu'il n'existe pas de préjudice moral (tout comme le tribunal dans le cas d'espèce). On peut donc émettre l'hypothèse bien fondée que les juges banquiers-juristes sont moins « punitifs » que les non banquiers-juristes. C'est la tendance inverse que l'on relève chez les juges issus du secteur du Bâtiment et Travaux publics (BTP).

En ce qui concerne le degré d'interventionnisme des juges dans les marchés (cas d'espèce $\mathrm{n}^{\circ}$ 2), on constate que les magistrats ayant fait du droit et appartenant au secteur de la banque et de la finance sont proportionnellement moins nombreux que l'ensemble des autres juges à répondre qu'ils auraient, dans des cas d'espèce 
similaires, pris la même décision que le tribunal - i.e. maintenu le contrat en vie contre la volonté d'une des parties. C'est à nouveau la tendance inverse que l'on relève chez les juges issus du secteur du BTP. Là encore, selon l'origine des juges, la sensibilité mobilisée dans ce type d'affaires n'est pas la même.

Tableau 1

Répartition des réponses aux trois cas d'espèce en fonction de caractéristiques socio-professionnelles des magistrats consulaires du Tribunal de commerce de Paris

\begin{tabular}{|c|c|c|c|c|}
\hline & $\begin{array}{c}\text { Juges, } \\
\text { banquiers et } \\
\text { juristes } 37 \\
\end{array}$ & $\begin{array}{l}\text { Juges issus } \\
\text { du BTP }\end{array}$ & Autres 38 & Ensemble \\
\hline \multirow[t]{2}{*}{ Effectif } & 24 & 13 & 114 & 151 \\
\hline & $\%$ & $\%$ & $\%$ & $\% \%$ \\
\hline \multicolumn{5}{|c|}{ Cas d'espèce $\mathrm{n}^{\circ} 1$ (punitivité) } \\
\hline $\begin{array}{l}\text { Évaluation identique du } \\
\text { préjudice matériel }\end{array}$ & 50 & 30,8 & 36,8 & 38,4 \\
\hline $\begin{array}{l}\text { Existence d'un préjudice } \\
\text { moral }\end{array}$ & 0 & 0 & 14,9 & 11,3 \\
\hline $\begin{array}{l}\text { Principe préjudice moral } \\
\text { d'une personne moral }\end{array}$ & 54,2 & 69,2 & 63,2 & 62,3 \\
\hline \multicolumn{5}{|c|}{ Cas d'espèce $n^{\circ} 2$ (interventionnisme dans les marchés) } \\
\hline Décision identique & 66,7 & 92,3 & 75,4 & 75,5 \\
\hline $\begin{array}{l}\text { Principe maintenir le } \\
\text { contrat en vie }\end{array}$ & 41,7 & 38,5 & 49,2 & 47,2 \\
\hline \multicolumn{5}{|c|}{ Cas d'espèce $\mathrm{n}^{\circ} 3$ (interventionnisme dans les conseils d'administration) } \\
\hline Décision identique report & 62,5 & 76,9 & 79,8 & 76,8 \\
\hline $\begin{array}{l}\text { Principe privilégier } \\
\text { l'intérêt social }\end{array}$ & 50 & 46,2 & 45,6 & 46,4 \\
\hline $\begin{array}{l}\text { Principe intervention du } \\
\text { juge dans la vie d'une } \\
\text { société }\end{array}$ & 29,2 & 30,8 & 17,6 & 20,5 \\
\hline
\end{tabular}

Enfin, en ce qui concerne le degré d'interventionnisme des juges dans les conseils d'administration (cas d'espèce $\mathrm{n}^{\circ} 3$ ), on constate que les trois quarts des magistrats consulaires auraient pris la même décision que le Président concernant la désignation d'un expert. Dans la même proportion, ils auraient donné leur chance aux minoritaires et ordonné le report de l'assemblée générale - sachant qu'une telle décision ne constitue pas pour autant une défense systématique des minoritaires. Mais lorsque les magistrats quittent le cas d'espèce pour aborder des principes plus généraux et répondre à la question de savoir si le juge doit privilégier l'intérêt social d'une société, i.e. sa survie, le consensus disparait. Pas loin de la moitié d'entre eux partage cette opinion ( $46 \%$ ), un quart y est hostile (22\%) et le reste hésite $(21 \%)$ ou refuse la question (10\%). On notera qu'avoir un diplôme en

37. À ne pas confondre avec l'ensemble des banquiers (y compris les banquiers non juristes) qui constituent $29 \%$ des juges du TCP (i.e. 47 juges).

38. Parmi les 114 juges inclus dans cette catégorie de contraste, 23 sont banquiers (mais non juristes), 34 proviennent du secteur des services aux entreprises, 12 des secteurs de la fabrication, 19 du secteur du commerce et de l'hôtellerie, et 26 du secteur industriel. 
droit clive les magistrats consulaires sur cette question ${ }^{39}$, par ailleurs - on l'a vu très facilement politisable.

En résumé, si l'on cherche à identifier des différences de sensibilités collectives ou de conventions, on trouve quelques déterminants des choix normatifs qui s'opposent au sein du Tribunal. Une première analyse, étayée par la lecture des entretiens, éclaire l'existence de ces sensibilités sectorielles. En effet, les juges à la fois juristes et issus du monde de la banque et de la finance tendent à adopter une attitude plus interventionniste que l'ensemble des juges dans le litige opposant les actionnaires d'une entreprise, en intra-organisationnel ; en cas de litige entre actionnaires, ils sont plus enclins à privilégier l'intérêt social d'une société (telle que défini par la direction et donc l'actionnaire majoritaire) que les autres répondants, et moins hostiles à l'intervention du juge dans la vie d'une société ${ }^{40}$. Ils ont en revanche une attitude beaucoup moins interventionniste que l'ensemble des juges dans le litige opposant les parties d'un contrat, sur un marché, en inter-organisationnel. Ils ont enfin une attitude moins "punitive» que l'ensemble des autres juges consulaires à l'égard de préjudices qualifiés de moraux et résultant de comportements de concurrence déloyale perturbant le fonctionnement supposé «naturel » des marchés.

C'est l'inverse qui est observé pour les juges issus du monde du BTP : ils ont tendance à adopter une attitude moins interventionniste que l'ensemble des juges dans le litige opposant les actionnaires d'une entreprise, en intra-organisationnel. Ils ont en revanche une attitude beaucoup plus interventionniste que l'ensemble des juges dans le litige opposant les parties d'un contrat sur un marché, en interorganisationnel. Ce contraste est confirmé par l'attitude moins punitive adoptée par les banquiers-juristes que par les juges issus du BTP à l'égard d'une demande recourant au préjudice moral d'une personne morale dans le fonctionnement des marchés. Rappelons aussi que les juges les plus jeunes ont tendance à être plus interventionnistes et punitifs que les plus anciens, à la fois dans les marchés et dans les entreprises.

Ces constats permettent, semble-t-il, de caractériser la régulation conjointe du monde des affaires par l'institution consulaire. Dans un contexte polynormatif, cette régulation conjointe apparaît ainsi comme le lieu d'une lutte d'influence pour la construction d'un cadre de référence commun indispensable à la qualification et à l'interprétation stabilisée des faits. Dans cette concurrence "épistémique " entre secteurs, celui de la banque et de la finance (même s'il ne s'est pas toujours organisé pour cela) est en position de promouvoir ses sensibilités et représentations collectives, des critères de justice et des conventions bien identifiables.

39. $57 \%$ (khi2 significatif) y sont hostiles.

40. L'un des juges, non banquier, émettait déjà cette hypothèse : les banquiers devraient être plus proches de l'intérêt social : « Parce que le banquier, lui aussi, a un intérêt dans l'intérêt social. Il a ses prêts qu'il veut voir remboursés. Donc, ils ont quand même une habitude systématique de favoriser l'intérêt social; d'ailleurs, la banque est toujours coincée entre le soutien abusif et la rupture abusive de crédits. " 


\section{Conclusion}

Notre approche du travail des juges consulaires met au jour une dimension peu lisible de la polynormativité contemporaine dans le contrôle social du monde des affaires. À partir de cas d'espèce réels, elle montre l'existence de variations correspondant à des luttes normatives ainsi qu'une relation entre carrière des juges, le milieu socioprofessionnel dont ils sont issus et les sensibilités et représentations collectives qu'ils mobilisent dans des affaires où ils/elles disposent d'un pouvoir souverain d'appréciation.

Mettre au jour ces sensibilités sectorielles correspond à la formulation d'hypothèses bien fondées sur le contrôle social du fonctionnement des marchés. Premièrement, nous montrons que les magistrats consulaires n'ont pas tous tendance à s'aligner sur les attitudes des juges juristes issus du secteur de la banque et de la finance. Même si ces derniers sont très centraux - voire dominants 41 - dans le processus d'apprentissage collectif du Tribunal, les autres juges mobilisent des sensibilités différentes.

Deuxièmement, cette étude apporte un contenu concret à la question du néocorporatisme dans les institutions de régulation conjointe du monde des affaires. La présence au Tribunal de commerce de Paris d'environ $29 \%$ de juges issus du secteur de la banque et de la finance soulevait cette question difficile, caractéristique - comme le soulignaient plusieurs juges eux-mêmes - des institutions consulaires. Les sensibilités mises au jour (interventionnisme et/ou punitivité plus ou moins forts de juges d'origines différentes dans le domaine des marchés ou dans le fonctionnement de la gouvernance d'entreprise) témoignent du sens que pourraient prendre des décisions de justice néo-corporatistes, donnant priorité à des intérêts particuliers sur l'intérêt général et exposant cette juridiction à des conflits d'intérêts systématiques - ou en tout cas à la suspicion générale issue de l'apparence de l'existence de ces conflits. Reste que des logiques transversales (dues au fonctionnement du Tribunal comme microcosme spécifique) viennent contrecarrer les logiques néo-corporatistes. La domination sans partage des banquiersjuristes ne semble pas acquise; elle ne s'impose en tout cas pas de manière très claire. Elle a affaire à une forte concurrence avec d'autres néo-corporatismes et avec des logiques transversales (intra-organisationnelles) d'articulation des conventions et des structures dans le partage des compétences.

La différence entre banque et BTP peut s'interpréter relativement aisément. Les banquiers-juristes n'ont pas, dans la plupart des cas, d'expérience directe des marchés et de leurs « coups bas "; ni d'intérêt direct dans les marchés. Ils ont en revanche une meilleure connaissance des conseils d'administration et un intérêt plus évident au contrôle de ce qui s'y passe - en particulier lorsque les entreprises sont leurs débitrices. C'est l'inverse que l'on s'attend à trouver chez les juges issus du BTP, un secteur caractérisé, entre autres, par une structure hiérarchique de sous-

41. Voir à ce sujet Emmanuel LAZEGA, Claire LEMERCIER et Lise Mounier, «A Spinning Top Model of Formal Structure and Informal Behaviour: Dynamics of Advice Networks in a Commercial Court ", European Management Review, 3, 2006, p. 113-122. 
traitances en cascade, structure qui engendre de nombreux contentieux entre partenaires de ces contrats de sous-traitance.

À l'heure du glissement des frontières entre privé et public, nous identifions un niveau de régulation conjointe des activités économiques en nous centrant sur le règlement des conflits commerciaux. Nous appuyant sur le cas du plus grand tribunal de commerce français, une institution consulaire, nous avons procédé en sociologues à une exploration descriptive de la diversité des conventions sur lesquelles cette institution s'appuie pour résoudre ces conflits. Ceci a permis de mieux comprendre certaines caractéristiques clé de la polynormativité et de la régulation conjointe comme forme de partage des responsabilités entre opérateurs privés et l'État (le pragmatisme juridique, la sélectivité sociale des magistrats dans leur recrutement, leur rapport au bénévolat de pouvoir, une culture de la consultation permettant le partage des compétences mais dominée par une petite élite de juges anciens et souvent banquiers-juristes). Nous pouvons à présent ajouter la coexistence de sensibilités et de représentations collectives différentes, indicateurs de néo-corporatisme.

Enfin, les limites de notre méthode imposent aussi une certaine prudence dans la généralisation des résultats. Ces derniers ne constituent pas un bilan des activités du TCP, bilan qui n'est pas envisageable sans accès à la base statistique du greffe du Tribunal. Au risque de surprendre, nous constatons enfin, malgré nos avancées dans la compréhension de ce qu'est la régulation conjointe des marchés, que le TCP reste à nos yeux une institution relativement opaque. À cet égard, nos explorations suggèrent qu'il y a certainement matière à promouvoir, au TCP comme ailleurs, une analyse des décisions des juges de l'économie au moyen d'une statistique des procès qui rendrait compte de la polynormativité à l'œuvre.

La connaissance des multiples dimensions de cette polynormativité et de cette régulation conjointe doit aboutir à une réflexion sur les limites de l'auto-régulation ${ }^{42}$ du monde des affaires et sur les nouveaux dispositifs de supervision que les États doivent mettre en place pour contrôler les processus régulatoires de type bottom-up ${ }^{43}$. Ces derniers s'appuient sur la production de représentations collectives et sur la mobilisation de ces représentations dans les raisonnements pratiques des acteurs de cette régulation conjointe ${ }^{44}$. Dans ce cadre, l'une des tâches de la sociologie économique et de la sociologie juridique est ainsi, nous semble-t-il, de continuer à rendre lisibles ces représentations et ces raisonnements qui, bien qu'hétérogènes et concurrents, ont légitimé depuis plusieurs siècles cette régulation conjointe - au risque de voir cette dernière devenir de plus en plus « disjointe »

42. Emmanuel LAZEGA, «Les conflits d'intérêts dans les cabinets américains d'avocats d'affaires : concurrence et auto-régulation", Sociologie du travail, 35, 1994, p. 315-336; ID., The Collegial Phenomenon: The Social Mechanisms of Cooperation Among Peers in a Corporate Law Partnership, Oxford, Oxford University Press, 2001 ; Dirk LeHMKUHL et Dieter RolofF, «Potential and Limits of Self-regulation : Private Actors in Theory and Practice», 2008, disponible à l'adresse Internet http://www.research-projects.uzh.ch/p4033.htm

43. Wesley CRAGG, «Ethics, Law and Corporate Self-Regulation », in ID. (eds.), Ethics Codes, Corporations and the Challenge of Globalization, Cheltenham (UK), Edward Elgar, 2005.

44. Emmanuel LAZEGA et Lise MoUNIER (avec la collaboration de Ana Maria FALCONI), Partage des compétences entre magistrats consulaires et usages du monde des affaires au Tribunal de commerce de Paris, op. cit. 
à mesure que le monde des affaires maîtrise le fonctionnement des institutions publiques qui exercent le contrôle social s'appliquant à lui-même. ${ }^{45}$.

\section{Les auteurs}

\section{Emmanuel LAZEGA}

Professeur de sociologie à l'Université Paris IX-Dauphine, membre de l'Irises-Cerso et membre associé du Centre Maurice Halbwachs (Cnrs-Ens-Ehess). Il travaille sur le contrôle social du monde des affaires du point de vue de la sociologie économique et de la sociologie du droit.

\section{Lise MOUNIER}

Sociologue, ingénieure de recherche au Centre Maurice Halbwachs (Cnrs-Ens-Ehess). Elle participe à des recherches mettant en œuvre l'analyse des réseaux sociaux.

45. Cette recherche a été réalisée avec le soutien de la Mission de recherche Droit et Justice et de l'Institut universitaire de France. Nous remercions les juges du Tribunal de commerce de Paris de leur accueil. Nous sommes reconnaissants aux Présidents du Tribunal, Monsieur Gilbert Costes et Madame Perrette Rey, d'avoir autorisé cette étude. Nous remercions Messieurs Roger Pujol et François Cambournac, Viceprésidents, et Monsieur Roland Schiff, Président de chambre au Tribunal de commerce de Paris ainsi que le Professeur Yves Chaput et Maîtres Yvon Martinet, Annabel Boccara et Arnaud Constant, avocats à la Cour, de leur aide et de leurs conseils pour la préparation de cette étude. Nous adressons nos remerciements à Ana Maria Falconi, Antonella Di Trani, Myrna Insua Belfer, Julie Laidi, Sylvan Lemaire, Claire Lemercier, Veronika Nagy, Ximena Perez Gonzales, Élodie Raux et Émilie Rogne pour leur participation à la réalisation des entretiens et à leur retranscription. Enfin, nous sommes reconnaissants à Ana Maria Falconi ainsi qu'aux évaluateurs anonymes de la revue pour leur relecture critique d'une première version de cet article. 